\title{
On Multi-step Estimation of Delay for SDE
}

\author{
YURY A. KUTOYANTS \\ Le Mans University, Le Mans, France \\ Tomsk State University, Tomsk, Russia
}

\begin{abstract}
We consider the problem of delay estimation by the observations of the solutions of several SDEs. It is known that the MLE for these models are consistent and asymptotically normal, but the likelihood ratio functions are not differentiable w.r.t. parameter and therefore numerical calculation of the MLEs has certain difficulties. We propose One-step and Two-step MLE, whose calculation has no such problems and provide estimator asymptotically equivalent to the MLE. These constructions are realized in two or three steps. First we construct preliminary estimators which are consistent and asymptotically normal, but not asymptotically efficient. Then we use these estimators and modified Fisher-score device to obtain One-step and Two-step MLEs. We suppose that its numerical realization is much more simple. Stochastic Pantograph equation is introduced and related statistical problems are discussed.
\end{abstract}

MSC 2000 Classification: 62G05, 62G20, 62M09.

Key words: One-step MLE, Two-step MLE, One-step MDE, stochastic Pantograph equation, delay estimation, asymptotic properties, asymptotic efficiency.

\section{Introduction}

We consider two types of models of stochastic differential equation (SDE) with delays, which can be illustrated by the following equations

$$
\begin{aligned}
& \mathrm{d} X_{t}=S\left(X_{t-\vartheta}\right) \mathrm{d} t+\varepsilon \mathrm{d} W_{t}, \quad X_{s}=x_{0}, \quad s \leq 0, \quad 0 \leq t \leq T, \\
& \mathrm{~d} X_{t}=S\left(X_{\vartheta t}\right) \mathrm{d} t+\varepsilon \mathrm{d} W_{t}, \quad X_{0}=x_{0}, \quad, \quad 0 \leq t \leq T .
\end{aligned}
$$


We call delay in the equation (1) shift-type and in the equation (2) scaletype. Here the functions $S(x)$ are known and smooth and parameter (delay) $\vartheta \in \Theta$. The set $\Theta=(\alpha, \beta), 0<\alpha<\beta<T$ in the case of equation (1) and $\Theta=(\alpha, \beta), 0<\alpha<\beta<1$ in the case of equation (2). The parameter $\vartheta$ has to be estimated by observations $X^{T}=\left(X_{t}, 0 \leq t \leq T\right)$ and the properties of estimators are studied in the asymptotic of small noise, i.e., as $\varepsilon \rightarrow 0$. Such perturbed dynamical systems were studied in [4] and different statistical problems related to such models can be found in [13]. We suppose that the function $S(x)$ is Lipschitz

$$
|S(x)-S(y)| \leq L|x-y|
$$

Under this condition the equations (1) and (2) have unique strong solutions and all polynomial moments of the solutions are finite (see Theorem 4.6 in [19]).

Ordinary and stochastic differential equations with delay (feedback with delay) are widely used in many applied sciences (see [2] (medicine), [3], [24], [25] (physics), [7] (neuron science), [9], [21], (population dynamics) and references therein). The same time statistical inference (estimation and testing concerning delays) for these systems does not attract sufficiently attention of the statisticians.

The possible difficulties in the study of these models of observations we illustrate with the help of the linear Ornstein-Uhlenbeck process with delay

$$
\mathrm{d} X_{t}=-\gamma X_{t-\vartheta} \mathrm{d} t+\sigma \mathrm{d} W_{t}, \quad X_{s}=x_{0}, s \leq 0, \quad 0 \leq t \leq T .
$$

Consider the problem of estimation $\vartheta \in \Theta$ by continuous time observations $X^{T}=\left(X_{t}, 0 \leq t \leq T\right)$ from (3). We suppose that the values $\gamma>0$ and $\sigma>0$ are known.

Let us write the equation (3) in integral form and change the variables $t \rightarrow t-\vartheta$. Then we obtain the representation

$$
X_{t-\vartheta}=x_{0}-\gamma \int_{0}^{t-\vartheta} X_{s-\vartheta} \mathrm{d} s+\sigma W_{t-\vartheta}, \quad t \geq \vartheta
$$

We see that the drift $\left.S\left(X_{t-\vartheta}\right)\right)=-\gamma X_{t-\vartheta}$ in the equation (3) is as smooth w.r.t. $\vartheta$ as the Wiener process w.r.t. time $t$, i.e., even the first derivative of the drift $\left.\partial S\left(X_{t-\vartheta}\right)\right) / \partial \vartheta$ does not exist. The following question naturally arises: does the problem of estimation $\vartheta$ by observations (4) regular (family of measures is LAN, Fisher information is finite), or singular (family of measures is not LAN, Fisher information is infinite)? What is amusing with this model of observations, we have the both cases depending on the type of asymptotics. 
If the time of observations is fixed and $\sigma \rightarrow 0$, then the problem of estimation is regular, family of measures is LAN and MLE $\hat{\vartheta}_{\sigma}$ is asymptotically normal

$$
\sigma^{-1}\left(\hat{\vartheta}_{\sigma}-\vartheta\right) \Longrightarrow \mathcal{N}\left(0, \mathrm{I}(\vartheta)^{-1}\right), \quad \mathrm{I}(\vartheta)=\gamma^{4} \int_{\vartheta}^{T} x_{t-2 \vartheta}^{2} \mathrm{~d} t
$$

(see [12], [13]). Here I $(\vartheta)$ plays the role of Fisher information and $x_{t}=x_{t}(\vartheta)$ is solution of the limit $(\sigma=0)$ equation

$$
\frac{\mathrm{d} x_{t}}{\mathrm{~d} t}=-\gamma x_{t-\vartheta}, \quad x_{s}=x_{0}, s \leq 0, \quad 0 \leq t \leq T .
$$

Note that the observations $X_{t}$ on the interval $[0, \vartheta]$ has no any information about the value of $\vartheta$ that is why the derivative of $-\gamma X_{t-\vartheta}=-\gamma x_{0}$ w.r.t. $\vartheta$ is zero.

If we fix $\sigma$ and consider asymptotic $T \rightarrow \infty$, then the problem of estimation becomes singular, family of measures is no more LAN and MLE $\hat{\vartheta}_{T}$ has another (non Gaussian) limit distribution

$$
T c\left(\hat{\vartheta}_{T}-\vartheta\right) \Longrightarrow \arg \sup _{u \in \mathcal{R}}\left(W(u)-\frac{|u|}{2}\right) .
$$

Here $c>0$ is some constant and $W(\cdot)$ is two-sided Wiener process [10], [14]. Recall that the similar properties has MLE $\hat{\vartheta}_{T}$ in the case of observations

$$
\mathrm{d} X_{t}=-\gamma \operatorname{sgn}\left(X_{t}-\vartheta\right) \mathrm{d} t+\sigma \mathrm{d} W_{t}, \quad X_{s}=x_{0}, s \leq 0, \quad 0 \leq t \leq T
$$

with discontinuous drift coefficient [14]. Hence here delay is equivalent to discontinuity (change-point in space) in the model. This is the only known to us model, which is the same time regular and singular (statistically) depending on the type of limit. The proofs are based on the powerful general results in [8].

The properties of the MLE $\hat{\vartheta}_{\varepsilon}$ for the model (1) were described in the works [12] (linear case) and in [1] (generalization of [12] to nonlinear case), see as well [13]. It was shown that the MLE is consistent, asymptotically normal

$$
\varepsilon^{-1}\left(\hat{\vartheta}_{\varepsilon}-\vartheta\right) \Longrightarrow \mathcal{N}\left(0, \mathrm{I}(\vartheta)^{-1}\right), \quad \mathrm{I}(\vartheta)=\int_{\vartheta}^{T} S^{\prime}\left(x_{t-\vartheta}\right)^{2} S\left(x_{t-2 \vartheta}\right)^{2} \mathrm{~d} t
$$

and asymptotically efficient.

The likelihood ratio function for the model (1) is

$$
L\left(\vartheta, X^{T}\right)=\exp \left\{\int_{0}^{T} \frac{S\left(X_{t-\vartheta}\right)}{\varepsilon^{2}} \mathrm{~d} X_{t}-\int_{0}^{T} \frac{S\left(X_{t-\vartheta}\right)^{2}}{2 \varepsilon^{2}} \mathrm{~d} t\right\}, \quad \vartheta \in \Theta
$$


and the MLE $\hat{\vartheta}_{\varepsilon}$ is defined by the equation

$$
L\left(\hat{\vartheta}_{\varepsilon}, X^{T}\right)=\sup _{\vartheta \in \Theta} L\left(\vartheta, X^{T}\right) .
$$

As the function $L\left(\vartheta, X^{T}\right)$ is not differentiable w.r.t. $\theta$ the realization of numerical algorithms of calculation $\hat{\vartheta}_{\varepsilon}$ can have certain difficulties.

The goal of this work is to construct estimators, whose calculation has no such difficulties and which have the same asymptotic properties as the MLE for the both models. This will be realized in two or three steps. First we construct some preliminary estimators, which have sufficiently good rate of convergence, but not asymptotically efficient. Then using these estimators and Fisher-score device we obtain One-step MLEs $\vartheta_{\varepsilon}^{\star}$. It is shown that the estimators $\vartheta_{\varepsilon}^{\star}$ are consistent and have the same asymptotic variance as the MLEs. The Fisher-score improvement of the preliminary estimators is a well-known approach in statistics and we do not give here the review of the corresponding literature. The discussion of the works related with diffusion processes can be found in [17].

Recall that the realization of Fisher-score device requires two derivatives of the trend coefficient w.r.t. unknown parameter. For example, suppose that the observed process is

$$
\mathrm{d} X_{t}=S(\vartheta, t) \mathrm{d} t+\varepsilon \mathrm{d} W_{t}, \quad X_{0}=0, \quad 0 \leq t \leq T
$$

and we have preliminary estimator $\bar{\vartheta}_{\varepsilon}$ such that $\varphi_{\varepsilon}^{-1}\left(\bar{\vartheta}_{\varepsilon}-\vartheta_{0}\right)$ is bounded in probability. Then we can write the One-step MLE formally as follows

$$
\vartheta_{\varepsilon}^{\star}=\bar{\vartheta}_{\varepsilon}+\int_{0}^{T} \frac{\dot{S}\left(\bar{\vartheta}_{\varepsilon}, t\right)}{\mathrm{I}\left(\bar{\vartheta}_{\varepsilon}\right)}\left[\mathrm{d} X_{t}-S\left(\bar{\vartheta}_{\varepsilon}, t\right) \mathrm{d} t\right], \quad \mathrm{I}(\vartheta)=\int_{0}^{T} \dot{S}(\vartheta, t)^{2} \mathrm{~d} t .
$$

Hence, using Taylor formula

$$
S\left(\vartheta_{0}, t\right)-S\left(\bar{\vartheta}_{\varepsilon}, t\right)=-\left(\bar{\vartheta}_{\varepsilon}-\vartheta_{0}\right) \dot{S}\left(\bar{\vartheta}_{\varepsilon}, t\right)-\frac{1}{2}\left(\bar{\vartheta}_{\varepsilon}-\vartheta_{0}\right)^{2} \ddot{S}\left(\tilde{\vartheta}_{\varepsilon}, t\right)
$$

we obtain the representation

$$
\begin{aligned}
\frac{\vartheta_{\varepsilon}^{\star}-\vartheta_{0}}{\varepsilon}= & \varepsilon^{-1}\left(\bar{\vartheta}_{\varepsilon}-\vartheta_{0}\right)+\mathrm{I}\left(\bar{\vartheta}_{\varepsilon}\right)^{-1} \int_{0}^{T} \dot{S}\left(\bar{\vartheta}_{\varepsilon}, t\right) \mathrm{d} W_{t} \\
& +\int_{0}^{T} \frac{\dot{S}\left(\bar{\vartheta}_{\varepsilon}, t\right)\left[S\left(\vartheta_{0}, t\right)-S\left(\bar{\vartheta}_{\varepsilon}, t\right)\right]}{\varepsilon \mathrm{I}\left(\bar{\vartheta}_{\varepsilon}\right)} \mathrm{d} t \\
= & \int_{0}^{T} \frac{\dot{S}(\vartheta, t)}{\mathrm{I}\left(\vartheta_{0}\right)} \mathrm{d} W_{t}-\frac{\left(\bar{\vartheta}_{\varepsilon}-\vartheta_{0}\right)^{2}}{\varepsilon} \int_{0}^{T} \frac{\dot{S}\left(\vartheta_{0}, t\right) \ddot{S}\left(\vartheta_{0}, t\right)}{2 \mathrm{I}\left(\vartheta_{0}\right)} \mathrm{d} t+o(1) .
\end{aligned}
$$


We see that if the seond derivative is bounded and $\varepsilon^{-1} \varphi_{\varepsilon}^{2} \rightarrow 0$, then the One-step MLE is asymptotically normal and asymptotically efficient. We say formally because there is the problem of definition of stochastic integral. We remind this example to show that in One-step MLE construction we need second derivative. The stochastic models considered in the present work have no even the first derivatives and we have to avoid the similar problem with stochastic integral too.

Statistical problems of parameter estimation related to different generalizations of the model (3) with the asymptotic $\sigma=\varepsilon \rightarrow 0$ were treated in the works [1], [12], [13] (see review in [15]). Some statistical problems for the related SDE with delay in the case of asymptotic $T \rightarrow \infty$ were studied in the works [6], [10], [15].

\section{Shift type-delay}

As it was mentioned above the problem of delay estimation we solve in two steps. First we construct a preliminary estimator, which is consistent and asymptotically normal and then we use this estimator and Fisher-score device to construct One-step MLE, which is asymptotically equivalent to the MLE. In the next section we propose and study such preliminary estimator.

MDE. We have observations $X^{T}=\left(X_{t}, 0 \leq t \leq T\right)$ of the solution of equation (1) and we have to estimate $\vartheta \in \Theta=(\alpha, \beta), 0<\alpha<\beta<T$. The true value we denote as $\vartheta_{0}$. The solution $x_{t}$ of the equation (5) for $t>\vartheta$ is a function of $\vartheta$ and we write it as $x_{t}(\vartheta)$.

The MDE $\bar{\vartheta}_{\varepsilon}$ we define as solution of the following equation

$$
\left\|X-x\left(\bar{\vartheta}_{\varepsilon}\right)\right\|=\inf _{\vartheta \in \Theta}\|X-x(\vartheta)\| .
$$

Here $\|\cdot\|$ is $L_{2}[\alpha, T]$-norm

$$
\|X-x(\vartheta)\|=\left(\int_{\alpha}^{T}\left[X_{t}-x_{t}(\vartheta)\right]^{2} \mathrm{~d} t\right)^{1 / 2} .
$$

Introduce Gaussian process $x_{t}^{(1)}=x_{t}^{(1)}\left(\vartheta_{0}\right)$ as solution of the equation

$$
\mathrm{d} x_{t}^{(1)}=S^{\prime}\left(x_{t-\vartheta_{0}}\right) x_{t-\vartheta_{0}}^{(1)} \mathrm{d} t+\mathrm{d} W_{t}, \quad x_{0}=0, \quad 0 \leq t \leq T .
$$

Note that $x_{t}^{(1)}=\partial X_{t} /\left.\partial \varepsilon\right|_{\varepsilon=0}$ (see [13]) and that $x_{t-\vartheta_{0}}^{(1)}=0,0 \leq t \leq \vartheta_{0}$. Hence $x_{t}^{(1)}=W_{t}, 0 \leq t \leq \vartheta_{0}$. The derivative $\dot{x}_{t}(\vartheta), t \geq \vartheta$ can be calculated as 
follows

$$
\dot{x}_{t}(\vartheta)=\frac{\partial x_{t}(\vartheta)}{\partial \vartheta}=-\int_{\vartheta}^{t} S^{\prime}\left(x_{s-\vartheta}\right) S\left(x_{s-2 \vartheta}\right) \mathrm{d} s .
$$

Introduce as well the Gaussian random variable

$$
\zeta\left(\vartheta_{0}\right)=\left(\int_{\vartheta_{0}}^{T} \dot{x}_{t}\left(\vartheta_{0}\right)^{2} \mathrm{~d} t\right)^{-1} \int_{\vartheta_{0}}^{T} x_{t}^{(1)}\left(\vartheta_{0}\right) \dot{x}_{t}\left(\vartheta_{0}\right) \mathrm{d} t \sim \mathcal{N}\left(0, \mathrm{D}\left(\vartheta_{0}\right)\right) .
$$

The variance $\mathrm{D}\left(\vartheta_{0}\right)$ of it can be calculated with the help of the equation (9), but its particular value is not important .

Theorem 1 Suppose that the function $S(x), x \in \mathcal{R}$ is positive, has two continuous bounded derivatives and there exists $x_{*} \in\left(x_{0}, x_{T-\beta}\right)$ such that $S^{\prime}\left(x_{*}\right) \neq 0$. Then the MDE is consistent, asymptotically normal

$$
\varepsilon^{-1}\left(\bar{\vartheta}_{\varepsilon}-\vartheta_{0}\right) \Longrightarrow \mathcal{N}\left(0, \mathrm{D}\left(\vartheta_{0}\right)\right)
$$

and for any $p>0$ the moments converge

$$
\varepsilon^{-p} \mathbf{E}_{\vartheta_{0}}\left|\bar{\vartheta}_{\varepsilon}-\vartheta_{0}\right|^{p} \longrightarrow \mathbf{E}_{\vartheta_{0}}\left|\zeta\left(\vartheta_{0}\right)\right|^{p}
$$

Proof. First we verify the consistency of MDE.

Lemma 1 For any $\nu>0$

$$
\sup _{\vartheta_{0} \in \Theta} \mathbf{P}_{\vartheta_{0}}\left(\left|\bar{\vartheta}_{\varepsilon}-\vartheta_{0}\right|>\nu\right) \longrightarrow 0 .
$$

Proof. The proof follows standard arguments used in such problems

$$
\begin{gathered}
\mathbf{P}_{\vartheta_{0}}\left(\left|\bar{\vartheta}_{\varepsilon}-\vartheta_{0}\right|>\nu\right)=\mathbf{P}_{\vartheta_{0}}\left(\inf _{\left|\vartheta-\vartheta_{0}\right|<\nu}\|X-x(\vartheta)\|>\inf _{\left|\vartheta-\vartheta_{0}\right| \geq \nu}\|X-x(\vartheta)\|\right) \\
\leq \mathbf{P}_{\vartheta_{0}}\left(\inf _{\left|\vartheta-\vartheta_{0}\right|<\nu}\left(\left\|X-x\left(\vartheta_{0}\right)\right\|+\left\|x\left(\vartheta_{0}\right)-x(\vartheta)\right\|\right)\right. \\
\left.>\inf _{\left|\vartheta-\vartheta_{0}\right| \geq \nu}\left(\left\|x\left(\vartheta_{0}\right)-x(\vartheta)\right\|-\left\|X-x\left(\vartheta_{0}\right)\right\|\right)\right) \\
=\mathbf{P}_{\vartheta_{0}}\left(2\left\|X-x\left(\vartheta_{0}\right)\right\|>\inf _{\left|\vartheta-\vartheta_{0}\right| \geq \nu}\left\|x\left(\vartheta_{0}\right)-x(\vartheta)\right\|\right) .
\end{gathered}
$$

Here we used the properties of norm $\|a+b\| \leq\|a\|+\|b\|,\|a+b\| \geq\|a\|-\|b\|$ and obvious equality $\inf _{\left|\vartheta-\vartheta_{0}\right|<\nu}\left\|x\left(\vartheta_{0}\right)-x(\vartheta)\right\|=0$. 
Introduce the function

$$
g\left(\vartheta_{0}, \nu\right)=\inf _{\left|\vartheta-\vartheta_{0}\right| \geq \nu}\left\|x\left(\vartheta_{0}\right)-x(\vartheta)\right\|
$$

and show that for any $\nu>0$ the function $g\left(\vartheta_{0}, \nu\right)$ satisfies $\inf _{\vartheta_{0} \in \Theta} g\left(\vartheta_{0}, \nu\right)>$ 0 . Suppose that $g\left(\vartheta_{0}, \nu\right)=0$, then there exist $\vartheta_{1}$ and $\vartheta_{0}$ such that $\left|\vartheta_{1}-\vartheta_{0}\right| \geq$ $\nu$ and $\left\|x\left(\vartheta_{0}\right)-x\left(\vartheta_{1}\right)\right\|=0$. The functions $x_{t}\left(\vartheta_{0}\right), x_{t}\left(\vartheta_{1}\right), 0 \leq t \leq T$ are continuous therefore we obtain equality $x_{t}\left(\vartheta_{0}\right)=x_{t}\left(\vartheta_{1}\right), 0 \leq t \leq T$. Hence for $\vartheta_{1}>\theta_{0}$ we have equality

$$
x_{t}\left(\vartheta_{1}\right)-x_{t}\left(\vartheta_{0}\right)=\left(\vartheta_{1}-\vartheta_{0}\right) \dot{x}_{t}(\tilde{\vartheta}) \equiv 0, \quad 0 \leq t \leq T,
$$

where $\vartheta_{0} \leq \tilde{\vartheta} \leq \vartheta_{1}$. As the function $S(x)$ is strictly positive, this equality implies (see (10)) $S^{\prime}\left(x_{s-\tilde{\vartheta}}\right) \equiv 0$ for all $s \in\left[\vartheta_{0} \leq s \leq T\right]$. It is possible to take such $s$ that $s-\tilde{\vartheta}=x_{*}$, but by condition of Theorem $S^{\prime}\left(x_{s-\tilde{\vartheta}}\right)=S^{\prime}\left(x_{*}\right) \neq 0$. Hence $g\left(\vartheta_{0}, \nu\right)>0$.

By Tchebychev inequality

$$
\mathbf{P}_{\vartheta_{0}}\left(\left|\bar{\vartheta}_{\varepsilon}-\vartheta_{0}\right|>\nu\right) \leq \frac{4}{g\left(\vartheta_{0}, \nu\right)^{2}} \mathbf{E}_{\vartheta_{0}} \int_{\alpha}^{T}\left[X_{t}-x_{t}\left(\vartheta_{0}\right)\right]^{2} \mathrm{~d} t \leq \frac{C \varepsilon^{2}}{g\left(\vartheta_{0}, \nu\right)^{2}} \rightarrow 0 .
$$

Here we used the estimate (31) proved in Appendix below. Therefore (12) is proved.

To prove the asymptotic normality (11) we note that the estimator $\bar{\vartheta}_{\varepsilon}$ satisfies the minimum distance equation (MDEq)

$$
\int_{\alpha}^{T}\left[X_{t}-x_{t}\left(\bar{\vartheta}_{\varepsilon}\right)\right] \dot{x}_{t}\left(\bar{\vartheta}_{\varepsilon}\right) \mathrm{d} t=0
$$

Let us introduce the random variable $\bar{u}_{\varepsilon}=\varepsilon^{-1}\left(\bar{\vartheta}_{\varepsilon}-\vartheta_{0}\right)$ and write the expansion

$$
\begin{aligned}
& x_{t}\left(\bar{\vartheta}_{\varepsilon}\right)=x_{t}\left(\vartheta_{0}+\varepsilon \bar{u}_{\varepsilon}\right)=x_{t}\left(\vartheta_{0}\right)+\varepsilon \bar{u}_{\varepsilon} \dot{x}_{t}\left(\vartheta_{0}\right)+O\left(\varepsilon^{2}\right), \\
& \dot{x}_{t}\left(\bar{\vartheta}_{\varepsilon}\right)=\dot{x}_{t}\left(\vartheta_{0}\right)+O(\varepsilon) .
\end{aligned}
$$

Then the MDEq allows us to write

$$
\int_{\alpha}^{T} \varepsilon^{-1}\left(X_{t}-x_{t}\left(\vartheta_{0}\right)\right)\left[\dot{x}_{t}\left(\vartheta_{0}\right)+O(\varepsilon)\right] \mathrm{d} t=\bar{u}_{\varepsilon} \int_{\alpha}^{T} \dot{x}_{t}\left(\vartheta_{0}\right)^{2} \mathrm{~d} t(1+O(\varepsilon))
$$

and

$$
\bar{u}_{\varepsilon}=\left(\int_{\alpha}^{T} \dot{x}_{t}\left(\vartheta_{0}\right)^{2} \mathrm{~d} t\right)^{-1} \int_{\alpha}^{T} \varepsilon^{-1}\left(X_{t}-x_{t}\left(\vartheta_{0}\right)\right) \dot{x}_{t}\left(\vartheta_{0}\right) \mathrm{d} t .
$$


Here

$$
\varepsilon^{-1}\left(X_{t}-x_{t}\left(\vartheta_{0}\right)\right) \longrightarrow x_{t}^{(1)}\left(\vartheta_{0}\right)
$$

in probability and therefore

$$
\bar{u}_{\varepsilon}=\varepsilon^{-1}\left(\bar{\vartheta}_{\varepsilon}-\vartheta_{0}\right) \Longrightarrow \zeta\left(\vartheta_{0}\right) .
$$

Moreover, it is possible to show that we have asymptotic normality in probability, because the limit random variable $\zeta\left(\vartheta_{0}\right)$ is defined on the same probability space.

For $\left|\vartheta-\vartheta_{0}\right| \leq \nu$ and sufficiently small $\nu>0$ we have

$$
\begin{aligned}
\left\|x(\vartheta)-x\left(\vartheta_{0}\right)\right\|^{2} & =\left(\vartheta-\vartheta_{0}\right)^{2}\|\dot{x}(\tilde{\vartheta})\|^{2}=\left(\vartheta-\vartheta_{0}\right)^{2}\left\|\dot{x}\left(\vartheta_{0}\right)\right\|^{2}(1+o(1)) \\
& \geq \frac{1}{2}\left(\vartheta-\vartheta_{0}\right)^{2}\left\|\dot{x}\left(\vartheta_{0}\right)\right\|^{2} .
\end{aligned}
$$

This estimate together with $g\left(\vartheta_{0}, \nu\right)>0$ allow us to write the estimate: there exists $\kappa>0$ such that

$$
g\left(\vartheta_{0}, \nu\right) \geq \kappa \nu .
$$

Let us put $\nu=\varepsilon y>0$, then for any integer $N>1$

$$
\begin{aligned}
\mathbf{P}_{\vartheta_{0}}\left(\varepsilon^{-1}\left|\bar{\vartheta}_{\varepsilon}-\vartheta_{0}\right|>y\right) \leq \frac{2^{2 N}}{\kappa^{2 N} y^{2 N}} \mathbf{E}_{\vartheta_{0}}\left|\int_{\alpha}^{T}\right| X_{t}-\left.\left.x_{t}\left(\vartheta_{0}\right)\right|^{2} \mathrm{~d} t\right|^{N} \\
\leq \frac{2^{2 N}(T-\alpha)^{N-1}}{\varepsilon^{2 N} \kappa^{2 N} y^{2 N}} \int_{\alpha}^{T} \mathbf{E}_{\vartheta_{0}}\left|X_{t}-x_{t}\left(\vartheta_{0}\right)\right|^{2 N} \mathrm{~d} t \leq \frac{C_{N}}{y^{2 N}},
\end{aligned}
$$

where the constant $C>0$ does not depend on $\varepsilon$ and $\vartheta_{0}$.

Let us denote $F_{\varepsilon}\left(\vartheta_{0}, y\right)=\mathbf{P}_{\vartheta_{0}}\left(\left|\bar{u}_{\varepsilon}\right|<y\right)$. Then for any $p>0$ we take $2 N \geq p+1$ and can write

$$
\begin{aligned}
\mathbf{E}_{\vartheta_{0}}\left|\frac{\bar{\vartheta}_{\varepsilon}-\vartheta_{0}}{\varepsilon}\right|^{p} & =\int_{0}^{\infty} y^{p} \mathrm{~d} F_{\varepsilon}\left(\vartheta_{0}, y\right)=1-\int_{1}^{\infty} y^{p} \mathrm{~d}\left(1-F_{\varepsilon}\left(\vartheta_{0}, y\right)\right) \\
& \leq 2+p \int_{1}^{\infty} y^{p-1}\left(1-F_{\varepsilon}\left(\vartheta_{0}, y\right)\right) \mathrm{d} y \\
& =2+p \int_{1}^{\infty} y^{p-1} \mathbf{P}_{\vartheta_{0}}\left(\varepsilon^{-1}\left|\bar{\vartheta}_{\varepsilon}-\vartheta_{0}\right|>y\right) \mathrm{d} y \\
& \leq 2+p C_{p} \int_{1}^{\infty} y^{p-1-2 N} \mathrm{~d} y \leq C .
\end{aligned}
$$

Therefore for any $p>1$ the random variables $\varepsilon^{-p}\left|\bar{\vartheta}_{\varepsilon}-\vartheta_{0}\right|^{p}$ are uniformly integrable and we obtain the convergence of moments (12). 
Remark that if the value $x_{*}$ mentioned in the Theorem 1 does not exist and $S^{\prime}(x)=0$ for all $x \in\left[x_{0}, x_{T-\beta}\right]$, then the function $S(x), x \in\left[x_{0}, x_{T-\beta}\right]$ does not depend on $x$ and consistent estimation of $\vartheta_{0}$ is impossible.

One-step MLE. Let us denote $\left\{\mathbf{P}_{\vartheta}^{(\varepsilon)}, \vartheta \in \Theta\right\}$ the family of measures induced on the measurable space $\left(\mathcal{C}_{(0, T)}, \mathscr{B}\right)$ of continuous on $[0, T]$ functions by the solutions of the equation (1) with different $\vartheta \in \Theta$. These measures are equivalent with the likelihood ratio function $L\left(\vartheta, X^{T}\right)$ [19]. Introduce the normalized likelihood ratio process

$$
Z_{\varepsilon}(u)=\frac{L\left(\vartheta_{0}+\varepsilon u, X^{T}\right)}{L\left(\vartheta_{0}, X^{T}\right)}, \quad u \in \mathbb{U}_{\varepsilon}=\left(\frac{\alpha-\vartheta_{0}}{\varepsilon}, \frac{\beta-\vartheta_{0}}{\varepsilon}\right) .
$$

We have (see (6))

$$
\begin{aligned}
\ln Z_{\varepsilon}(u)= & \int_{0}^{T} \frac{S\left(X_{t-\vartheta_{0}-\varepsilon u}\right)-S\left(X_{t-\vartheta_{0}}\right)}{\varepsilon} \mathrm{d} W_{t} \\
& -\int_{0}^{T} \frac{\left[S\left(X_{t-\vartheta_{0}-\varepsilon u}\right)-S\left(X_{t-\vartheta_{0}}\right)\right]^{2}}{2 \varepsilon^{2}} \mathrm{~d} t .
\end{aligned}
$$

As it follows from the given below proof of the theorem (see also [1]), this likelihood ratio admits the representation

$$
Z_{\varepsilon}(u)=\exp \left\{-u \int_{\vartheta_{0}}^{T} S^{\prime}\left(x_{t-\vartheta_{0}}\right) S\left(x_{t-2 \vartheta_{0}}\right) \mathrm{d} W_{t}-\frac{u^{2}}{2} \mathrm{I}\left(\vartheta_{0}\right)+o(1)\right\} .
$$

Here $x_{t-\vartheta_{0}}=x_{t-\vartheta_{0}}\left(\vartheta_{0}\right), x_{t-2 \vartheta_{0}}=x_{t-2 \vartheta_{0}}\left(\vartheta_{0}\right)$ and I $(\vartheta)$ plays the role of Fisher information. It is given by the equality

$$
\mathrm{I}(\vartheta)=\int_{\vartheta}^{T} S^{\prime}\left(x_{t-\vartheta}(\vartheta)\right)^{2} S\left(x_{t-2 \vartheta}(\vartheta)\right)^{2} \mathrm{~d} t .
$$

As

$$
\int_{\vartheta_{0}}^{T} S^{\prime}\left(x_{t-\vartheta_{0}}\right) S\left(x_{t-2 \vartheta_{0}}\right) \mathrm{d} W_{t} \sim \mathcal{N}\left(0, \mathrm{I}\left(\vartheta_{0}\right)\right)
$$

the family of measures $\left\{\mathbf{P}_{\vartheta}^{(\varepsilon)}, \vartheta \in \Theta\right\}$ is locally asymptotically normal (LAN) for all $\vartheta_{0} \in \Theta$. Therefore we have Hajek-Le Cam's lower bound on the mean squared risk of all estimators $\vartheta_{\varepsilon}$

$$
\lim _{\nu \rightarrow 0} \lim _{\varepsilon \rightarrow 0} \sup _{\left|\vartheta-\vartheta_{0}\right| \leq \nu} \varepsilon^{-2} \mathbf{E}_{\vartheta}\left(\vartheta_{\varepsilon}-\vartheta\right)^{2} \geq \mathrm{I}\left(\vartheta_{0}\right)^{-1}
$$


As usual, we call estimator $\vartheta_{\varepsilon}^{*}$ asymptotically efficient if for this estimator we have equality

$$
\lim _{\nu \rightarrow 0} \lim _{\varepsilon \rightarrow 0} \sup _{\left|\vartheta-\vartheta_{0}\right| \leq \nu} \varepsilon^{-2} \mathbf{E}_{\vartheta}\left(\vartheta_{\varepsilon}^{*}-\vartheta\right)^{2}=\mathrm{I}\left(\vartheta_{0}\right)^{-1}
$$

for all $\vartheta_{0} \in \Theta$. Recall that the MLE $\hat{\vartheta}_{\varepsilon}$ for this model of observations is asymptotically efficient [1], [13].

One-step MLE $\vartheta_{\varepsilon}^{\star}$ for this model of observations formally can be written as follows

$$
\begin{aligned}
\vartheta_{\varepsilon}^{\star} & =\bar{\vartheta}_{\varepsilon}+\left.\varepsilon^{2} \mathrm{I}\left(\bar{\vartheta}_{\varepsilon}\right)^{-1} \frac{\partial \ln L\left(\vartheta, X^{T}\right)}{\partial \vartheta}\right|_{\vartheta=\bar{\vartheta}_{\varepsilon}} \\
& =\bar{\vartheta}_{\varepsilon}+\mathrm{I}\left(\bar{\vartheta}_{\varepsilon}\right)^{-1} \int_{0}^{T} S^{\prime}\left(X_{t-\bar{\vartheta}_{\varepsilon}}\right) \frac{\partial X_{t-\bar{\vartheta}_{\varepsilon}}}{\partial \vartheta}\left[\mathrm{d} X_{t}-S\left(X_{t-\bar{\vartheta}_{\varepsilon}}\right) \mathrm{d} t\right] .
\end{aligned}
$$

We say formally because in this writing there are two difficulties. The first one: as the estimator $\bar{\vartheta}_{\varepsilon}$ depends on all observations on the interval $[\alpha, T]$ the Itô stochastic integral is not well defined. The second problem, of course, is the calculation of the derivative $\partial X_{t-\bar{\vartheta}_{\varepsilon}} / \partial \vartheta$. Our goal is to find an alternative expression for One-step MLE which does not have such problems.

Let us write the expression for $X_{t-\vartheta}$, without Wiener part

$$
\hat{X}_{t-\vartheta}=x_{0}+\int_{0}^{t-\vartheta} S\left(X_{s-\vartheta_{0}}\right) \mathrm{d} s .
$$

Then

$$
\frac{\partial \hat{X}_{t-\vartheta}}{\partial \vartheta}=-S\left(X_{t-\vartheta-\vartheta}\right) \mathbb{1}_{\{t>\vartheta\}}
$$

First we replace the stochastic process $X_{t-\bar{\vartheta}_{\varepsilon}}$ by $x_{t-\bar{\vartheta}_{\varepsilon}}\left(\bar{\vartheta}_{\varepsilon}\right)$ and write the integral as follows

$$
\begin{aligned}
& \left.\int_{0}^{T} S^{\prime}\left(x_{t-\bar{\vartheta}_{\varepsilon}}\right) \frac{\partial x_{t-\vartheta}\left(\bar{\vartheta}_{\varepsilon}\right)}{\partial \vartheta}\right|_{\vartheta=\bar{\vartheta}_{\varepsilon}}\left[\mathrm{d} X_{t}-S\left(X_{t-\bar{\vartheta}_{\varepsilon}}\right) \mathrm{d} t\right] \\
& \quad=\int_{\bar{\vartheta}_{\varepsilon}}^{T} S^{\prime}\left(x_{t-\bar{\vartheta}_{\varepsilon}}\right) S\left(x_{t-2 \bar{\vartheta}_{\varepsilon}}\right) S\left(X_{t-\bar{\vartheta}_{\varepsilon}}\right) \mathrm{d} t-\int_{\bar{\vartheta}_{\varepsilon}}^{T} S^{\prime}\left(x_{t-\bar{\vartheta}_{\varepsilon}}\right) S\left(x_{t-2 \bar{\vartheta}_{\varepsilon}}\right) \mathrm{d} X_{t} .
\end{aligned}
$$

Note that $\left.X_{t-\vartheta}\right|_{\varepsilon=0}=x_{t-\vartheta}\left(\vartheta_{0}\right)$ and we differentiate on $\vartheta$ only, but then replace the second value $\vartheta_{0}$ by $\bar{\vartheta}_{\varepsilon}$ too. Remind that the likelihood ratio depends on the true value $\vartheta_{0}$ but we never differentiate it on $\vartheta_{0}$. 
Further, let us denote

$$
H(t, \vartheta)=S^{\prime}\left(x_{t-\vartheta}(\vartheta)\right) S\left(x_{t-2 \vartheta}(\vartheta)\right) .
$$

Then

$$
\mathrm{d}\left[H(t, \vartheta) X_{t}\right]=H(t, \vartheta) \mathrm{d} X_{t}+X_{t} H_{t}^{\prime}(t, \vartheta) \mathrm{d} t
$$

and

$$
\int_{\vartheta}^{T} S^{\prime}\left(x_{t-\vartheta}\right) S\left(x_{t-2 \vartheta}\right) \mathrm{d} X_{t}=H(T, \vartheta) X_{T}-H(\vartheta, \vartheta) X_{\vartheta}-\int_{\vartheta}^{T} H_{t}^{\prime}(t, \vartheta) X_{t} \mathrm{~d} t .
$$

Of course, we have

$$
\begin{aligned}
H_{t}^{\prime}(t, \vartheta)=S^{\prime \prime}\left(x_{t-\vartheta}(\vartheta)\right) & S\left(x_{t-2 \vartheta}(\vartheta)\right)^{2} \\
+ & S^{\prime}\left(x_{t-\vartheta}(\vartheta)\right) S^{\prime}\left(x_{t-2 \vartheta}(\vartheta)\right) S\left(x_{t-3 \vartheta}(\vartheta)\right) \mathbb{1}_{\{t \geq 2 \vartheta\}} .
\end{aligned}
$$

Now we can put

$$
\Psi\left(\bar{\vartheta}_{\varepsilon}\right)=H\left(T, \bar{\vartheta}_{\varepsilon}\right) X_{T}-H\left(\bar{\vartheta}_{\varepsilon}, \bar{\vartheta}_{\varepsilon}\right) X_{\bar{\vartheta}_{\varepsilon}}-\int_{\bar{\vartheta}_{\varepsilon}}^{T} H_{t}^{\prime}\left(t, \bar{\vartheta}_{\varepsilon}\right) X_{t} \mathrm{~d} t
$$

and define the One-step MLE

$$
\vartheta_{\varepsilon}^{\star}=\bar{\vartheta}_{\varepsilon}+\mathrm{I}\left(\bar{\vartheta}_{\varepsilon}\right)^{-1}\left[\int_{\bar{\vartheta}_{\varepsilon}}^{T} S^{\prime}\left(x_{t-\bar{\vartheta}_{\varepsilon}}\right) S\left(x_{t-2 \bar{\vartheta}_{\varepsilon}}\right) S\left(X_{t-\bar{\vartheta}_{\varepsilon}}\right) \mathrm{d} t-\Psi\left(\bar{\vartheta}_{\varepsilon}\right)\right] .
$$

Its properties are given in the next theorem.

Theorem 2 Suppose that the function $S(\cdot)$ is positive, has four continuous bounded derivatives and there exists $x_{*} \in\left(x_{0}, x_{T-\beta}\right)$ such that $S^{\prime}\left(x_{*}\right) \neq 0$. Then the One-step MLE $\vartheta_{\varepsilon}^{\star}$ is consistent, asymptotically normal

$$
\varepsilon^{-1}\left(\vartheta_{\varepsilon}^{\star}-\vartheta_{0}\right) \Longrightarrow \mathcal{N}\left(0, \mathrm{I}\left(\vartheta_{0}\right)^{-1}\right) \text {. }
$$

and asymptotically efficient.

Proof. Let us study the expression

$$
\begin{aligned}
R\left(\bar{\vartheta}_{\varepsilon}\right)= & \int_{\bar{\vartheta}_{\varepsilon}}^{T} S^{\prime}\left(x_{t-\bar{\vartheta}_{\varepsilon}}\right) S\left(x_{t-2 \bar{\vartheta}_{\varepsilon}}\right) S\left(X_{t-\bar{\vartheta}_{\varepsilon}}\right) \mathrm{d} t-\Psi\left(\bar{\vartheta}_{\varepsilon}\right)=H\left(\bar{\vartheta}_{\varepsilon}, \bar{\vartheta}_{\varepsilon}\right) X_{\bar{\vartheta}_{\varepsilon}} \\
& +\int_{\bar{\vartheta}_{\varepsilon}}^{T}\left[H\left(t, \bar{\vartheta}_{\varepsilon}\right) S\left(X_{t-\bar{\vartheta}_{\varepsilon}}\right)-H_{t}^{\prime}\left(t, \bar{\vartheta}_{\varepsilon}\right) X_{t}\right] \mathrm{d} t-H\left(T, \bar{\vartheta}_{\varepsilon}\right) X_{T} .
\end{aligned}
$$


We have to expand it at the vicinity of the point $\vartheta_{0}$. As $\bar{\vartheta}_{\varepsilon}=\vartheta_{0}+\varepsilon \bar{u}_{\varepsilon}$ and the function $H(t, \vartheta)$ is sufficiently smooth w.r.t. $\vartheta$ and $t$ we can write the expansions for the function $H(\cdot)$. Before we note that there is a problem with expansion of the term $H\left(\bar{\vartheta}_{\varepsilon}, \bar{\vartheta}_{\varepsilon}\right) X_{\bar{\vartheta}_{\varepsilon}}$. The process $X_{t}$ has no derivative but we have

$$
\begin{aligned}
X_{\bar{\vartheta}_{\varepsilon}} & =X_{\vartheta_{0}}+\int_{\vartheta_{0}}^{\bar{\vartheta}_{\varepsilon}} S\left(X_{t-\vartheta_{0}}\right) \mathrm{d} t+\varepsilon\left[W_{\bar{\vartheta}_{\varepsilon}}-W_{\vartheta_{0}}\right] \\
& =X_{\vartheta_{0}}+\varepsilon \bar{u}_{\varepsilon} S\left(x_{0}\right)(1+o(1))+\varepsilon^{3 / 2} O(1) .
\end{aligned}
$$

Therefore we can write

$$
\begin{aligned}
H\left(T, \bar{\vartheta}_{\varepsilon}\right) & =H\left(T, \vartheta_{0}\right)+\varepsilon \bar{u}_{\varepsilon} H_{\vartheta}^{\prime}\left(T, \vartheta_{0}\right)+\varepsilon^{2} A\left(T, \tilde{\vartheta}_{\varepsilon}\right), \\
H\left(\bar{\vartheta}_{\varepsilon}, \bar{\vartheta}_{\varepsilon}\right) & =H\left(\vartheta_{0}, \vartheta_{0}\right)+\varepsilon \bar{u}_{\varepsilon}\left[H_{t}^{\prime}\left(\vartheta_{0}, \vartheta_{0}\right)+H_{\vartheta}^{\prime}\left(\vartheta_{0}, \vartheta_{0}\right)\right]+\varepsilon^{2} B\left(\tilde{\vartheta}_{\varepsilon}, \tilde{\vartheta}_{\varepsilon}\right),
\end{aligned}
$$

and for the integral we have

$$
\begin{aligned}
& \int_{\bar{\vartheta}_{\varepsilon}}^{T} H_{t}^{\prime}\left(t, \bar{\vartheta}_{\varepsilon}\right) X_{t} \mathrm{~d} t=\int_{\vartheta_{0}}^{T} H_{t}^{\prime}\left(t, \vartheta_{0}\right) X_{t} \mathrm{~d} t \\
& \quad+\varepsilon \bar{u}_{\varepsilon}\left[\int_{\bar{\vartheta}_{\varepsilon}}^{T} H_{t, \vartheta}^{\prime \prime}\left(t, \vartheta_{0}\right) X_{t} \mathrm{~d} t-H_{t}^{\prime}\left(\vartheta_{0}, \vartheta_{0}\right) X_{\vartheta_{0}}\right]+\varepsilon^{3 / 2} C\left(T, \tilde{\vartheta}_{\varepsilon}, \tilde{\vartheta}_{\varepsilon}\right) .
\end{aligned}
$$

Here we denoted $A(\cdot), B(\cdot)$ and $C(\cdot)$ the corresponding residuals. Note that all polynomial moments of these quantities are finite. Of course, the values of $\tilde{\vartheta}_{\varepsilon}$ in these three expansions are different and this is just a symbolical writing.

Therefore we have the presentation

$$
\Psi\left(\bar{\vartheta}_{\varepsilon}\right)=\Psi\left(\vartheta_{0}\right)+\varepsilon \bar{u}_{\varepsilon} \hat{\Psi}\left(\vartheta_{0}\right)+\varepsilon^{3 / 2} O(1),
$$

where

$$
\begin{aligned}
\Psi\left(\vartheta_{0}\right) & =\int_{\vartheta_{0}}^{T} S^{\prime}\left(x_{t-\vartheta_{0}}\right) S\left(x_{t-2 \vartheta_{0}}\right) \mathrm{d} X_{t} \\
& =\int_{\vartheta_{0}}^{T} H\left(t, \vartheta_{0}\right) S\left(X_{t-\vartheta_{0}}\right) \mathrm{d} t+\varepsilon \int_{\vartheta_{0}}^{T} H\left(t, \vartheta_{0}\right) \mathrm{d} W_{t}
\end{aligned}
$$


and

$$
\begin{aligned}
\hat{\Psi}\left(\vartheta_{0}\right)= & H_{\vartheta}^{\prime}\left(T, \vartheta_{0}\right) X_{T}-\left[H_{\vartheta}^{\prime}\left(\vartheta_{0}, \vartheta_{0}\right)+H_{t}^{\prime}\left(\vartheta_{0}, \vartheta_{0}\right)\right] X_{\vartheta_{0}}-H\left(\vartheta_{0}, \vartheta_{0}\right) S\left(x_{0}\right) \\
& \quad+H_{t}^{\prime}\left(\vartheta_{0}, \vartheta_{0}\right) X_{\vartheta_{0}}-\int_{\vartheta_{0}}^{T} H_{t, \vartheta}^{\prime \prime}\left(t, \vartheta_{0}\right) X_{t} \mathrm{~d} t \\
= & H_{\vartheta}^{\prime}\left(T, \vartheta_{0}\right) X_{T}-H_{\vartheta}^{\prime}\left(\vartheta_{0}, \vartheta_{0}\right) X_{\vartheta_{0}}-H\left(\vartheta_{0}, \vartheta_{0}\right) S\left(x_{0}\right) \\
& \quad-\int_{\vartheta_{0}}^{T} H_{t, \vartheta}^{\prime \prime}\left(t, \vartheta_{0}\right) X_{t} \mathrm{~d} t \\
= & \int_{\vartheta_{0}}^{T} H_{\vartheta}^{\prime}\left(t, \vartheta_{0}\right) \mathrm{d} X_{t}-H\left(\vartheta_{0}, \vartheta_{0}\right) S\left(x_{0}\right) \\
= & \int_{\vartheta_{0}}^{T} H_{\vartheta}^{\prime}\left(t, \vartheta_{0}\right) S\left(X_{t-\vartheta_{0}}\right) \mathrm{d} t+\varepsilon \int_{\vartheta_{0}}^{T} H_{\vartheta}^{\prime}\left(t, \vartheta_{0}\right) \mathrm{d} W_{t}-H\left(\vartheta_{0}, \vartheta_{0}\right) S\left(x_{0}\right) .
\end{aligned}
$$

Further

$$
\begin{aligned}
\int_{\bar{\vartheta}_{\varepsilon}}^{T} H\left(t, \bar{\vartheta}_{\varepsilon}\right) S\left(X_{t-\bar{\vartheta}_{\varepsilon}}\right) \mathrm{d} t= & \int_{\vartheta_{0}}^{T} H\left(t, \vartheta_{0}\right) S\left(X_{t-\bar{\vartheta}_{\varepsilon}}\right) \mathrm{d} t-\varepsilon \bar{u}_{\varepsilon} H\left(\vartheta_{0}, \vartheta_{0}\right) S\left(x_{0}\right) \\
& +\varepsilon \bar{u}_{\varepsilon} \int_{\vartheta_{0}}^{T} H_{\vartheta}^{\prime}\left(t, \vartheta_{0}\right) S\left(X_{t-\vartheta_{0}}\right) \mathrm{d} t+O\left(\varepsilon^{3 / 2}\right) .
\end{aligned}
$$

Therefore

$$
\begin{gathered}
R\left(\bar{\vartheta}_{\varepsilon}\right)=\int_{\vartheta_{0}}^{T} H\left(t, \vartheta_{0}\right)\left[S\left(X_{t-\bar{\vartheta}_{\varepsilon}}\right)-S\left(X_{t-\vartheta_{0}}\right)\right] \mathrm{d} t \\
-\varepsilon \int_{\vartheta_{0}}^{T} H\left(t, \vartheta_{0}\right) \mathrm{d} W_{t}+O\left(\varepsilon^{3 / 2}\right) \\
=-\varepsilon \bar{u}_{\varepsilon} \int_{\vartheta_{0}}^{T} H\left(t, \vartheta_{0}\right) S^{\prime}\left(x_{t-\vartheta_{0}}\right) S\left(x_{t-\vartheta_{0}}\right) \mathrm{d} t \\
\quad-\varepsilon \int_{\vartheta_{0}}^{T} H\left(t, \vartheta_{0}\right) \mathrm{d} W_{t}+O\left(\varepsilon^{3 / 2}\right) \\
=-\varepsilon \bar{u}_{\varepsilon} \mathrm{I}\left(\vartheta_{0}\right)-\varepsilon \int_{\vartheta_{0}}^{T} H\left(t, \vartheta_{0}\right) \mathrm{d} W_{t}+O\left(\varepsilon^{3 / 2}\right) .
\end{gathered}
$$

The obtained relations allow us to write

$$
\begin{aligned}
\frac{\vartheta_{\varepsilon}^{\star}-\vartheta_{0}}{\varepsilon} & =\bar{u}_{\varepsilon}-\mathrm{I}\left(\bar{\vartheta}_{\varepsilon}\right)^{-1} \int_{\vartheta_{0}}^{T} H\left(t, \vartheta_{0}\right) \mathrm{d} W_{t}-\bar{u}_{\varepsilon} \frac{\mathrm{I}\left(\vartheta_{0}\right)}{\mathrm{I}\left(\bar{\vartheta}_{\varepsilon}\right)}+O\left(\varepsilon^{1 / 2}\right) \\
& =-\mathrm{I}\left(\vartheta_{0}\right)^{-1} \int_{\vartheta_{0}}^{T} H\left(t, \vartheta_{0}\right) \mathrm{d} W_{t}\left(1+o\left(\varepsilon^{1 / 2}\right)\right)
\end{aligned}
$$


Recall that

$$
-\mathrm{I}\left(\vartheta_{0}\right)^{-1} \int_{\vartheta_{0}}^{T} H\left(t, \vartheta_{0}\right) \mathrm{d} W_{t} \sim \mathcal{N}\left(0, \mathrm{I}\left(\vartheta_{0}\right)^{-1}\right)
$$

Note that the Fisher information is separated from zero, i.e.,

$$
\inf _{\vartheta \in \Theta} I(\vartheta)>0
$$

and we can easily verify the convergence of all polynomial moments of $o\left(\varepsilon^{1 / 2}\right)$ above. Moreover, in all estimates of the type

$$
\mathbf{E}_{\vartheta_{0}}\left|o\left(\varepsilon^{1 / 2}\right)\right|^{p} \leq C \varepsilon^{\frac{p}{2}}
$$

with any $p>0$ and corresponding constants $C=C(K)>0$ not depending on $\vartheta_{0} \in K$ for any compact set $K \subset \Theta$. This provides us the uniform convergence of the moments of $u_{\varepsilon}^{\star}=\varepsilon^{-1}\left(\vartheta_{\varepsilon}^{\star}-\vartheta_{0}\right)$. For the second moment we have

$$
\sup _{\left|\vartheta-\vartheta_{0}\right| \leq \nu} \mathbf{E}_{\vartheta}\left|\frac{\vartheta_{\varepsilon}^{\star}-\vartheta}{\varepsilon}\right|^{2} \stackrel{\varepsilon \rightarrow 0}{\longrightarrow} \sup _{\left|\vartheta-\vartheta_{0}\right| \leq \nu} \mathrm{I}(\vartheta)^{-1} \stackrel{\nu \rightarrow 0}{\longrightarrow} \mathrm{I}\left(\vartheta_{0}\right)^{-1} .
$$

Therefore the One-step MLE $\vartheta_{\varepsilon}^{\star}$ is asymptotically efficient.

Example 1. Suppose that we have Ornstein-Uhlenbeck process with delay

$$
\mathrm{d} X_{t}=-\gamma X_{t-\vartheta} \mathrm{d} t+\varepsilon \mathrm{d} W_{t}, \quad X_{s}=x_{0}, \quad s \leq 0, \quad 0 \leq t \leq T .
$$

Here $\gamma>0$ is supposed to be known, $x_{0}>0$ and $\vartheta \in(\alpha, \beta), 0<\alpha<\beta<T$. The solution $x_{t}=x_{t}\left(\vartheta_{0}\right)$ of the limit equation

$$
\frac{\mathrm{d} x_{t}}{\mathrm{~d} t}=-\gamma x_{t-\vartheta_{0}}, \quad x_{s}=x_{0}, s \leq 0, \quad 0 \leq t \leq T
$$

can be written in explicit form as follows

$$
x_{t}\left(\vartheta_{0}\right)=x_{0} y_{t}\left(\vartheta_{0}\right)-\gamma x_{0} \int_{-\vartheta_{0}}^{0} y_{t-s-\vartheta_{0}}\left(\vartheta_{0}\right) \mathrm{d} s, \quad t \geq 0,
$$

where

$$
y_{t}\left(\vartheta_{0}\right)=\sum_{k=0}^{\left[\frac{t}{\vartheta_{0}}\right]}(-1)^{k} \frac{\gamma^{k}}{k !}\left(t-k \vartheta_{0}\right)^{k}
$$


$[a]$ is entire part of $a$ (see [11] for details).

The preliminary MDE $\bar{\vartheta}_{\varepsilon}$ is

$$
\bar{\vartheta}_{\varepsilon}=\arg \inf _{\alpha<\vartheta<\beta} \int_{\alpha}^{T}\left[X_{t}-x_{t}(\vartheta)\right]^{2} \mathrm{~d} t .
$$

Recall that the Fisher information in this problem is

$$
\mathrm{I}(\vartheta)=\gamma^{4} \int_{\vartheta}^{T} x_{t-2 \vartheta}(\vartheta)^{2} \mathrm{~d} t .
$$

The One-step MLE $\vartheta_{\varepsilon}^{\star}$ has the following form

$$
\begin{aligned}
\vartheta_{\varepsilon}^{\star}=\bar{\vartheta}_{\varepsilon}+\mathrm{I} & \left(\bar{\vartheta}_{\varepsilon}\right)^{-1}\left[\gamma^{2} x_{0} X_{\bar{\vartheta}_{\varepsilon}}-\gamma^{2} x_{T-2 \bar{\vartheta}_{\varepsilon}}\left(\bar{\vartheta}_{\varepsilon}\right) X_{T}\right. \\
& \left.-\gamma^{3} \int_{\bar{\vartheta}_{\varepsilon}}^{T} x_{t-2 \bar{\vartheta}_{\varepsilon}}\left(\bar{\vartheta}_{\varepsilon}\right) X_{t-\bar{\vartheta}_{\varepsilon}} \mathrm{d} t-\gamma^{3} \int_{2 \bar{\vartheta}_{\varepsilon}}^{T} x_{t-3 \bar{\vartheta}_{\varepsilon}}\left(\bar{\vartheta}_{\varepsilon}\right) X_{t} \mathrm{~d} t\right] .
\end{aligned}
$$

By Theorem 2 we have

$$
\varepsilon^{-1}\left(\vartheta_{\varepsilon}^{\star}-\vartheta_{0}\right) \Longrightarrow \mathcal{N}\left(0, \mathrm{I}(\vartheta)^{-1}\right)
$$

\section{Scale-type delay}

Below we consider the models of type (2). We start with the linear model with the equation, which can be called Stochastic Pantograph Equation and then we study the nonlinear model.

Stochastic Pantograph Equation. Recall that the deterministic Pantograph equation

$$
\frac{\mathrm{d} x_{t}}{\mathrm{~d} t}=a x_{t}+b x_{\vartheta t}, \quad x_{0}, \quad t \geq 0
$$

was introduced by Ockendon and Tayler in 1971 [23]. This equation describes the special construction on the electric locomotive of the British Railways, which allowed to collect the current from an overhead wire. Here the parameter $0<\vartheta<1$ defines the delay $d=t-\vartheta t$. The further study of this equation can be found in the thesis [5].

Introduce the condition

$\mathscr{A}$. The reals $x_{0} \neq 0, b \neq 0$ and $a+b \neq 0$. 
Note that if $x_{0}=0$, then the solution of (17) is $x_{t} \equiv 0$. If $b=0$, then there is no delay. If $a+b=0$, then we have once more a trivial solution $x_{t} \equiv x_{0}$.

The stochastic version of Pantograph equation can be

$$
\mathrm{d} X_{t}=a X_{t} \mathrm{~d} t+b X_{\vartheta t} \mathrm{~d} t+\varepsilon \mathrm{d} W_{t}, \quad X_{0}=x_{0}, \quad t \geq 0 .
$$

Consider the problem of estimation $\vartheta \in \Theta=(\alpha, \beta), 0<\alpha<\beta<1$ by the observations $X^{T}=\left(X_{t}, 0 \leq t \leq T\right)$. The reals $a, b \neq 0, x_{0} \neq 0$ and $\varepsilon \in(0,1]$ are supposed to be known. The likelihood ratio function is

$$
L\left(\vartheta, X^{T}\right)=\exp \left\{\int_{0}^{T} \frac{b X_{\vartheta t}}{\varepsilon^{2}} \mathrm{~d} X_{t}-\int_{0}^{T} \frac{\left[b^{2} X_{\vartheta t}^{2}-2 a b X_{t} X_{\vartheta t}\right]}{2 \varepsilon^{2}} \mathrm{~d} t\right\}, \quad \vartheta \in \Theta
$$

and numerical calculation of the MLE $\hat{\vartheta}_{\varepsilon}$ defined by the equation (7) has the same problems as in the case of observations (1). Note that the asymptotic $(\varepsilon \rightarrow 0)$ properties of the MLE in the case $a=0$ were described in [12]. Here we consider the construction of the one-step MLE using the Fisher-score device similar to that given above.

The role of Fisher information plays the quantity

$$
\mathrm{I}(\vartheta)=b^{2} \int_{0}^{T} t^{2}\left[a x_{\vartheta t}(\vartheta)+b x_{\vartheta^{2} t}(\vartheta)\right]^{2} \mathrm{~d} t .
$$

As preliminary estimator wa can take the MDE $\bar{\vartheta}_{\varepsilon}$ defined by the equation (8), where $x(\vartheta)=\left(x_{t}(\vartheta), 0 \leq t \leq T\right)$ is the solution of the equation (18). It can be shown that this estimator is consistent and asymptotically normal $\varepsilon^{-1}\left(\bar{\vartheta}_{\varepsilon}-\vartheta_{0}\right)$. Moreover, it is consistent and asymptotically normal even if we take observations $X^{\tau_{\varepsilon}}=\left(X_{t}, 0 \leq t \leq \tau_{\varepsilon}\right)$ on the interval $\left[0, \tau_{\varepsilon}\right]$ with $\tau_{\varepsilon} \rightarrow 0$.

Estimator of substitution. Note that the model (18) allows us to use another more simple estimator as preliminary one, which we introduce as follows

$$
\bar{\vartheta}_{\tau_{\varepsilon}}=\frac{2}{(a+b) b \tau_{\varepsilon}^{2}}\left[X_{\tau_{\varepsilon}}-x_{0}-x_{0} \tau_{\varepsilon}(a+b)\right]-\frac{a}{b} .
$$

Below we show that this is estimator of substitution.

Theorem 3 Let the condition $\mathscr{A}$ be fulfilled and $\tau_{\varepsilon}=\varepsilon^{\gamma}$, where $\gamma \in\left(\frac{2}{5}, \frac{2}{3}\right)$. Then the estimator $\bar{\vartheta}_{\tau_{\varepsilon}}$ is consistent and asymptotically normal

$$
\frac{\tau_{\varepsilon}^{3 / 2}}{\varepsilon}\left(\bar{\vartheta}_{\tau_{\varepsilon}}-\vartheta_{0}\right) \Longrightarrow \mathcal{N}\left(0, D^{2}\right), \quad D^{2}=\frac{4}{(a+b)^{2} b^{2}}
$$


Moreover we have the convergence of moments too: for any $p>0$

$$
\varepsilon^{-p\left(1-\frac{3}{2} \gamma\right)} \mathbf{E}_{\vartheta_{0}}\left|\left(\bar{\vartheta}_{\tau_{\varepsilon}}-\vartheta_{0}\right)\right|^{p} \longrightarrow D^{\frac{p}{2}} \mathbf{E}|\xi|^{p}, \quad \xi \sim \mathcal{N}(0,1) .
$$

Proof. The function $x_{t}(\vartheta)$ can be expanded at the vicinity of the point $t=0$

$$
\begin{aligned}
x_{t}(\vartheta) & =x_{0}+\int_{0}^{t}\left[a x_{s}+b x_{\vartheta s}\right] \mathrm{d} s \\
& =x_{0}+x_{0} t[a+b]+\frac{1}{2} x_{0} t^{2}[a+b][a+b \vartheta]+O\left(t^{3}\right)
\end{aligned}
$$

and we can write

$$
\vartheta_{\tau}=\frac{2}{(a+b) b \tau^{2}}\left[x_{\tau}(\vartheta)-x_{0}-x_{0} \tau(a+b)\right]-\frac{a}{b}+O(\tau) .
$$

Remind that the process $X_{t}$ admits the representation

$$
X_{t}=x_{t}\left(\vartheta_{0}\right)+\varepsilon x_{t}^{(1)}\left(\vartheta_{0}\right) \mathrm{d} t+O\left(\varepsilon^{2}\right)
$$

where the Gaussian process $x_{t}^{(1)}\left(\vartheta_{0}\right)$ (derivative of $X_{t}$ w.r.t. $\varepsilon$ ) has the stochastic differential

$$
\mathrm{d} x_{t}^{(1)}\left(\vartheta_{0}\right)=\left[a x_{t}^{(1)}\left(\vartheta_{0}\right)+b x_{\vartheta_{0} t}^{(1)}\left(\vartheta_{0}\right)\right] \mathrm{d} t+\mathrm{d} W_{t}, \quad x_{0}^{(1)}\left(\vartheta_{0}\right)=0
$$

and the expansion of it is

$$
x_{\tau_{\varepsilon}}^{(1)}\left(\vartheta_{0}\right)=W_{\tau_{\varepsilon}}+O\left(\tau_{\varepsilon}^{2}\right) .
$$

Hence

$$
\begin{aligned}
\bar{\vartheta}_{\tau_{\varepsilon}} & =\frac{2}{(a+b) b \tau_{\varepsilon}^{2}}\left[x_{\tau_{\varepsilon}}\left(\vartheta_{0}\right)-x_{0}-x_{0} \tau_{\varepsilon}(a+b)\right]-\frac{a}{b}+\frac{2 x_{\tau_{\varepsilon}}^{(1)}\left(\vartheta_{0}\right)}{(a+b) b} \frac{\varepsilon}{\tau_{\varepsilon}^{2}} \\
& =\vartheta_{0}+\frac{2 W_{\tau_{\varepsilon}}}{(a+b) b} \frac{\varepsilon}{\tau_{\varepsilon}^{2}}+O\left(\tau_{\varepsilon}\right)=\vartheta_{0}+\frac{2 W_{1}}{(a+b) b} \frac{\varepsilon}{\tau_{\varepsilon}^{3 / 2}}+O\left(\tau_{\varepsilon}\right) .
\end{aligned}
$$

Here $W_{1} \sim \mathcal{N}(0,1)$. Therefore

$$
\frac{\tau_{\varepsilon}^{3 / 2}}{\varepsilon}\left(\bar{\vartheta}_{\tau_{\varepsilon}}-\vartheta_{0}\right)=\frac{2 W_{1}}{(a+b) b}+O\left(\frac{\tau_{\varepsilon}^{5 / 2}}{\varepsilon}\right)
$$

and if we suppose that

$$
\frac{\tau_{\varepsilon}^{3 / 2}}{\varepsilon} \longrightarrow \infty, \quad \frac{\tau_{\varepsilon}^{5 / 2}}{\varepsilon} \longrightarrow 0,
$$


then we obtain the asymptotic normality (20). Note that the choice $\tau_{\varepsilon}=\varepsilon^{\gamma}$ with $\gamma \in\left(\frac{2}{5}, \frac{2}{3}\right)$ provides $(22)$.

The rate of convergence of this estimator is $\varepsilon^{\kappa}$ with $0<\kappa<\frac{2}{5}$, i.e.,

$$
\frac{\bar{\vartheta}_{\tau_{\varepsilon}}-\vartheta_{0}}{\varepsilon^{\kappa}} \Longrightarrow \mathcal{N}\left(0, D^{2}\right)
$$

This rate of convergence is not sufficient for the construction of the One-step MLE. We need an estimator with the rate $\kappa>\frac{1}{2}$. That is why we propose below another estimator which uses $\bar{\vartheta}_{\tau_{\varepsilon}}$ and has the rate of convergence up to the values grater than $\frac{1}{2}$.

One-step MDE. Let us consider the equation (8), where $X=X^{\tau_{\varepsilon}}=$ $\left(X_{t}, 0 \leq t \leq \tau_{\varepsilon}\right)$. The solution $\check{\vartheta}_{\tau_{\varepsilon}}$ of it satisfies the following minimum distance equation

$$
\int_{0}^{\tau_{\varepsilon}}\left[X_{t}-x_{t}\left(\check{\vartheta}_{\tau_{\varepsilon}}\right)\right] \dot{x}_{t}\left(\check{\vartheta}_{\tau_{\varepsilon}}\right) \mathrm{d} t=0
$$

Here $\dot{x}_{t}(\vartheta)=\partial x_{t}(\vartheta) / \partial \vartheta$ is solution of the equation

$$
\frac{\mathrm{d} \dot{x}_{t}(\vartheta)}{\mathrm{d} t}=a \dot{x}_{t}(\vartheta)+b \dot{x}_{\vartheta t}(\vartheta)+b t\left[a x_{\vartheta t}(\vartheta)+b x_{\vartheta^{2} t}(\vartheta)\right], \quad \dot{x}_{0}(\vartheta)=0 .
$$

Let us write $x_{t}\left(\check{\vartheta}_{\tau_{\varepsilon}}\right)=x_{t}\left(\vartheta_{0}\right)+\left(\check{\vartheta}_{\tau_{\varepsilon}}-\vartheta_{0}\right) \dot{x}_{t}\left(\tilde{\vartheta}_{\tau_{\varepsilon}}\right)$, where the value $\tilde{\vartheta}_{\tau_{\varepsilon}}$ satisfies the relation $\left|\tilde{\vartheta}_{\tau_{\varepsilon}}-\vartheta_{0}\right| \leq\left|\check{\vartheta}_{\tau_{\varepsilon}}-\vartheta_{0}\right|$. From the equation (24) we obtain the representation

$$
\check{\vartheta}_{\tau_{\varepsilon}}=\vartheta_{0}+\left(\int_{0}^{\tau_{\varepsilon}} \dot{x}_{t}\left(\tilde{\vartheta}_{\tau_{\varepsilon}}\right) \dot{x}_{t}\left(\check{\vartheta}_{\tau_{\varepsilon}}\right) \mathrm{d} t\right)^{-1} \int_{0}^{\tau_{\varepsilon}}\left[X_{t}-x_{t}\left(\vartheta_{0}\right)\right] \dot{x}_{t}\left(\check{\vartheta}_{\tau_{\varepsilon}}\right) \mathrm{d} t .
$$

Let us denote

$$
Q_{\tau_{\varepsilon}}(\vartheta)=\int_{0}^{\tau_{\varepsilon}} \dot{x}_{t}(\vartheta)^{2} \mathrm{~d} t
$$

The One-step MDE $\vartheta_{\tau_{\varepsilon}}^{*}$ we introduce as follows

$$
\vartheta_{\tau_{\varepsilon}}^{*}=\bar{\vartheta}_{\tau_{\varepsilon}}+Q_{\tau_{\varepsilon}}\left(\bar{\vartheta}_{\tau_{\varepsilon}}\right)^{-1} \int_{0}^{\tau_{\varepsilon}}\left[X_{t}-x_{t}\left(\bar{\vartheta}_{\tau_{\varepsilon}}\right)\right] \dot{x}_{t}\left(\bar{\vartheta}_{\tau_{\varepsilon}}\right) \mathrm{d} t
$$

Theorem 4 Let the conditions of Theorem 3 be fulfilled, then for any $p>0$ there exists a constant $C>0$ such that

$$
\varepsilon^{-p \delta} \mathbf{E}_{\vartheta_{0}}\left|\vartheta_{\tau_{\varepsilon}}^{*}-\vartheta_{0}\right|^{p} \leq C
$$

where $\delta \in\left(0, \frac{2}{3}\right)$. 
Proof. Let us write

$$
\frac{\vartheta_{\tau_{\varepsilon}}^{*}-\vartheta_{0}}{\varepsilon^{\delta}}=\frac{\bar{\vartheta}_{\tau_{\varepsilon}}-\vartheta_{0}}{\varepsilon^{\delta}}+\varepsilon^{-\delta} Q_{\tau_{\varepsilon}}\left(\bar{\vartheta}_{\tau_{\varepsilon}}\right)^{-1} \int_{0}^{\tau_{\varepsilon}}\left[X_{t}-x_{t}\left(\bar{\vartheta}_{\tau_{\varepsilon}}\right)\right] \dot{x}_{t}\left(\bar{\vartheta}_{\tau_{\varepsilon}}\right) \mathrm{d} t
$$

We have

$$
\varepsilon^{-\delta}\left[X_{t}-x_{t}\left(\bar{\vartheta}_{\tau_{\varepsilon}}\right)\right]=\varepsilon^{-\delta}\left[X_{t}-x_{t}\left(\vartheta_{0}\right)\right]-\varepsilon^{-\delta}\left(\bar{\vartheta}_{\tau_{\varepsilon}}-\vartheta_{0}\right) \dot{x}_{t}\left(\tilde{\vartheta}_{\tau_{\varepsilon}}\right) .
$$

Recall that

$$
\mathbf{E}_{\vartheta_{0}}\left|X_{t}-x_{t}\left(\vartheta_{0}\right)\right|^{p} \leq C \varepsilon^{p}
$$

(see (33) below). Therefore for any $\delta<1$ we have $\varepsilon^{-\delta}\left[X_{t}-x_{t}\left(\vartheta_{0}\right)\right]=o(1)$. Further, we can write once more the expansion $\dot{x}_{t}\left(\tilde{\vartheta}_{\tau_{\varepsilon}}\right)=\dot{x}_{t}\left(\bar{\vartheta}_{\tau_{\varepsilon}}\right)+\left(\tilde{\vartheta}_{\tau_{\varepsilon}}-\right.$ $\left.\bar{\vartheta}_{\tau_{\varepsilon}}\right) \ddot{x}_{t}\left(\tilde{\bar{\vartheta}}_{\tau_{\varepsilon}}\right)$. Hence

$$
\begin{aligned}
\varepsilon^{-\delta} Q_{\tau_{\varepsilon}}\left(\bar{\vartheta}_{\tau_{\varepsilon}}\right)^{-1} \int_{0}^{\tau_{\varepsilon}}\left[X_{t}-x_{t}\left(\bar{\vartheta}_{\tau_{\varepsilon}}\right)\right] \dot{x}_{t}\left(\bar{\vartheta}_{\tau_{\varepsilon}}\right) \mathrm{d} t \\
=o(1)-\varepsilon^{-\delta}\left(\bar{\vartheta}_{\tau_{\varepsilon}}-\vartheta_{0}\right) Q_{\tau_{\varepsilon}}\left(\bar{\vartheta}_{\tau_{\varepsilon}}\right)^{-1} \int_{0}^{\tau_{\varepsilon}} \dot{x}_{t}\left(\bar{\vartheta}_{\tau_{\varepsilon}}\right)^{2} \mathrm{~d} t \\
\quad+\varepsilon^{-\delta}\left(\bar{\vartheta}_{\tau_{\varepsilon}}-\vartheta_{0}\right)\left(\bar{\vartheta}_{\tau_{\varepsilon}}-\tilde{\vartheta}_{\tau_{\varepsilon}}\right) Q_{\tau_{\varepsilon}}\left(\bar{\vartheta}_{\tau_{\varepsilon}}\right)^{-1} \int_{0}^{\tau_{\varepsilon}} \ddot{x}_{t}\left(\tilde{\bar{\vartheta}}_{\tau_{\varepsilon}}\right) \dot{x}_{t}\left(\bar{\vartheta}_{\tau_{\varepsilon}}\right) \mathrm{d} t \\
=o(1)-\frac{\left(\bar{\vartheta}_{\tau_{\varepsilon}}-\vartheta_{0}\right)}{\varepsilon^{\delta}}+\frac{\left(\bar{\vartheta}_{\tau_{\varepsilon}}-\vartheta_{0}\right)\left(\bar{\vartheta}_{\tau_{\varepsilon}}-\tilde{\vartheta}_{\tau_{\varepsilon}}\right)}{\varepsilon^{\delta}} \frac{\int_{0}^{\tau_{\varepsilon}} \ddot{x}_{t}\left(\tilde{\bar{\vartheta}}_{\tau_{\varepsilon}}\right) \dot{x}_{t}\left(\bar{\vartheta}_{\tau_{\varepsilon}}\right) \mathrm{d} t}{Q_{\tau_{\varepsilon}}\left(\bar{\vartheta}_{\tau_{\varepsilon}}\right)}
\end{aligned}
$$

For the small values of $t$ the solution of the equation (25) can be written as follows

$$
\dot{x}_{t}(\vartheta)=\frac{1}{2} b t^{2} x_{0}(a+B)+O\left(t^{3}\right) .
$$

For the second derivative we have the similar expansions

$$
\ddot{x}_{t}(\vartheta)=\frac{1}{2} a t^{2} x_{0}(a+b)+\frac{1}{2} b t^{2} x_{0}(a+b)+O\left(t^{3}\right)
$$

Hence we have estimates

$$
Q_{\tau_{\varepsilon}}\left(\bar{\vartheta}_{\tau_{\varepsilon}}\right) \geq c \tau_{\varepsilon}^{5}, \quad \int_{0}^{\tau_{\varepsilon}} \ddot{x}_{t}\left(\tilde{\bar{\vartheta}}_{\tau_{\varepsilon}}\right) \dot{x}_{t}\left(\bar{\vartheta}_{\tau_{\varepsilon}}\right) \mathrm{d} t \leq C \tau_{\varepsilon}^{5} .
$$

Note as well that

$$
\left|\left(\bar{\vartheta}_{\tau_{\varepsilon}}-\vartheta_{0}\right)\left(\bar{\vartheta}_{\tau_{\varepsilon}}-\tilde{\vartheta}_{\tau_{\varepsilon}}\right)\right| \leq 2\left|\bar{\vartheta}_{\tau_{\varepsilon}}-\vartheta_{0}\right|^{2} .
$$


Therefore we obtain the relation

$$
\left|\frac{\left(\bar{\vartheta}_{\tau_{\varepsilon}}-\vartheta_{0}\right)\left(\bar{\vartheta}_{\tau_{\varepsilon}}-\tilde{\vartheta}_{\tau_{\varepsilon}}\right)}{\varepsilon^{\delta}} \frac{\int_{0}^{\tau_{\varepsilon}} \ddot{x}_{t}\left(\tilde{\bar{\vartheta}}_{\tau_{\varepsilon}}\right) \dot{x}_{t}\left(\bar{\vartheta}_{\tau_{\varepsilon}}\right) \mathrm{d} t}{Q_{\tau_{\varepsilon}}\left(\bar{\vartheta}_{\tau_{\varepsilon}}\right)}\right| \leq C \frac{\left|\bar{\vartheta}_{\tau_{\varepsilon}}-\vartheta_{0}\right|^{2}}{\varepsilon^{\delta}}=\frac{\varepsilon^{2 \kappa}}{\varepsilon^{\delta}}\left|\bar{u}_{\varepsilon}\right|^{2}
$$

where $\bar{u}_{\varepsilon}=\varepsilon^{-\kappa}\left(\bar{\vartheta}_{\tau_{\varepsilon}}-\vartheta_{0}\right)$. If we take $\delta<2 \kappa$, i.e., $0<\delta<\frac{2}{3}$, then for the One-step MDE we obtain

$$
\frac{\vartheta_{\tau_{\varepsilon}}^{*}-\vartheta_{0}}{\varepsilon^{\delta}}=o(1), \quad \mathbf{E}_{\vartheta_{0}}\left|\frac{\vartheta_{\tau_{\varepsilon}}^{*}-\vartheta_{0}}{\varepsilon^{\delta}}\right|^{p}=o(1)
$$

Now the rate of convergence of preliminary estimator $\vartheta_{\tau_{\varepsilon}}^{*}$ can be grater than $\frac{1}{2}$ and it can be used for the construction of One-step MLE.

Two-step MLE. Having this preliminary estimator we construct the Two-step MLE as follows

$$
\vartheta_{\varepsilon}^{\star}=\vartheta_{\tau_{\varepsilon}}^{*}+\mathrm{I}_{\tau_{\varepsilon}}\left(\vartheta_{\tau_{\varepsilon}}^{*}\right)^{-1} b \int_{\tau_{\varepsilon}}^{T} t\left[a x_{t}+b x_{\vartheta_{\tau_{\varepsilon}}^{*}}\right]\left[\mathrm{d} X_{t}-\left(a X_{t}+b X_{\vartheta_{\tau_{\varepsilon}}^{*}}\right) \mathrm{d} t\right],
$$

where

$$
\mathrm{I}_{\tau}(\vartheta)=b^{2} \int_{\tau}^{T} t^{2}\left[a x_{\vartheta t}(\vartheta)+b x_{\vartheta^{2} t}(\vartheta)\right]^{2} \mathrm{~d} t .
$$

Note that we have no more problem with definition of the stochastic integral because the estimator $\vartheta_{\tau_{\varepsilon}}^{*}$ depends on the observations $X^{\tau_{\varepsilon}}=\left(X_{t}, 0 \leq t \leq \tau_{\varepsilon}\right)$ and the integral starts at the moment $\tau_{\varepsilon}$.

Theorem 5 Suppose that the condition $\mathscr{A}$ is fulfilled and $\tau_{\varepsilon}=\varepsilon^{\gamma}$ with $\gamma \in$ $\left(\frac{2}{5}, \frac{1}{2}\right)$. Then the estimator $\vartheta_{\varepsilon}^{\star}$ is consistent, asymptotically normal

$$
\varepsilon^{-1}\left(\vartheta_{\varepsilon}^{\star}-\vartheta_{0}\right) \Longrightarrow \mathcal{N}\left(0, \mathrm{I}\left(\vartheta_{0}\right)^{-1}\right)
$$

and we have the convergence of polynomial moments.

Proof. Below we substitute the observations (18), where $\vartheta=\vartheta_{0}$, in the stochastic integral

$$
\begin{aligned}
\varepsilon^{-1}\left(\vartheta_{\varepsilon}^{\star}-\vartheta_{0}\right)=\varepsilon^{-1} & \left(\vartheta_{\tau_{\varepsilon}}^{*}-\vartheta_{0}\right)+\mathrm{I}_{\tau_{\varepsilon}}\left(\vartheta_{\tau_{\varepsilon}}^{*}\right)^{-1} b \int_{\tau_{\varepsilon}}^{T} t\left[a x_{t}+b x_{\vartheta_{\tau_{\varepsilon}}} t\right] \mathrm{d} W_{t} \\
& +\varepsilon^{-1} \mathrm{I}_{\tau_{\varepsilon}}\left(\vartheta_{\tau_{\varepsilon}}^{*}\right)^{-1} b^{2} \int_{\tau_{\varepsilon}}^{T} t\left[a x_{t}+b x_{\vartheta_{\tau_{\varepsilon}}^{*} t}\right]\left(X_{\vartheta_{0} t}-X_{\vartheta_{\tau_{\varepsilon}}^{*} t}\right) \mathrm{d} t
\end{aligned}
$$


From the consistency of the estimator $\vartheta_{\tau_{\varepsilon}}^{*}$ and continuity of the corresponding functions we obtain the convergences

$$
b^{2} \int_{\tau_{\varepsilon}}^{T} t^{2}\left[a x_{t}+b x_{\vartheta_{\tau_{\varepsilon}}^{*}}\right]^{2} \mathrm{~d} t \longrightarrow \mathrm{I}\left(\vartheta_{0}\right), \quad \mathrm{I}_{\tau_{\varepsilon}}\left(\vartheta_{\tau_{\varepsilon}}^{*}\right) \longrightarrow \mathrm{I}\left(\vartheta_{0}\right)
$$

Hence by the central limit theorem for stochastic integrals we have the asymptotic normality

$$
\mathrm{I}_{\tau_{\varepsilon}}\left(\vartheta_{\tau_{\varepsilon}}^{*}\right)^{-1} b \int_{\tau_{\varepsilon}}^{T} t\left[a x_{t}+b x_{\vartheta_{\tau_{\varepsilon}}^{*}}\right] \mathrm{d} W_{t} \Longrightarrow \mathcal{N}\left(0, \mathrm{I}\left(\vartheta_{0}\right)^{-1}\right)
$$

Remark, that for this model of observations we have the asymptotic normality "in probability" too

$$
\mathrm{I}_{\tau_{\varepsilon}}\left(\vartheta_{\tau_{\varepsilon}}^{*}\right)^{-1} b \int_{\tau_{\varepsilon}}^{T} t\left[a x_{t}+b x_{\vartheta_{\tau_{\varepsilon}}^{*} t}\right] \mathrm{d} W_{t} \longrightarrow \mathrm{I}\left(\vartheta_{0}\right)^{-1} b \int_{0}^{T} t\left[a x_{t}+b x_{\vartheta_{0} t}\right] \mathrm{d} W_{t},
$$

where

$$
\mathrm{I}\left(\vartheta_{0}\right)^{-1} b \int_{0}^{T} t\left[a x_{t}+b x_{\vartheta_{0} t}\right] \mathrm{d} W_{t} \sim \mathcal{N}\left(0, \mathrm{I}\left(\vartheta_{0}\right)^{-1}\right)
$$

Further, uniformly on $t \in[0, T]$

$$
\begin{array}{r}
X_{\vartheta_{\tau_{\varepsilon}}^{*} t}-X_{\vartheta_{0} t}=x_{\vartheta_{\tau_{\varepsilon}}^{*} t}\left(\vartheta_{0}\right)-x_{\vartheta_{0} t}\left(\vartheta_{0}\right)+\varepsilon\left[x_{\vartheta_{\tau_{\varepsilon}}^{*} t}^{(1)}\left(\vartheta_{0}\right)-x_{\vartheta_{0} t}^{(1)}\left(\vartheta_{0}\right)\right]+O\left(\varepsilon^{2}\right) \\
=\left(\vartheta_{\tau_{\varepsilon}}^{*}-\vartheta_{0}\right) t\left[a x_{\vartheta_{0} t}+b x_{\vartheta_{0}^{2} t}\right]+\varepsilon \Delta_{\varepsilon}+O\left(\varepsilon^{2}\right)+O\left(\left(\vartheta_{\tau_{\varepsilon}}^{*}-\vartheta_{0}\right)^{2}\right),
\end{array}
$$

where

$$
\begin{aligned}
\Delta_{\varepsilon} & =x_{\vartheta_{\tau_{\varepsilon}}^{*} t}^{(1)}\left(\vartheta_{0}\right)-x_{\vartheta_{0} t}^{(1)}\left(\vartheta_{0}\right) \\
& =\int_{\vartheta_{0} t}^{\vartheta_{\tau_{\varepsilon}}^{*} t}\left[a x_{s}^{(1)}\left(\vartheta_{0}\right)+b x_{\vartheta_{0} s}^{(1)}\left(\vartheta_{0}\right)\right] \mathrm{d} s+W_{\vartheta_{\tau_{\varepsilon}}^{*} t}-W_{\vartheta_{0} t} \\
& =O\left(\vartheta_{\tau_{\varepsilon}}^{*}-\vartheta_{0}\right)+O\left(\left|\vartheta_{\tau_{\varepsilon}}^{*}-\vartheta_{0}\right|^{1 / 2}\right)=O\left(\left|\vartheta_{\tau_{\varepsilon}}^{*}-\vartheta_{0}\right|^{1 / 2}\right) .
\end{aligned}
$$

Therefore

$$
X_{\vartheta_{\tau_{\varepsilon}}^{*} t}-X_{\vartheta_{0} t}=\left(\vartheta_{\tau_{\varepsilon}}^{*}-\vartheta_{0}\right) t\left[a x_{\vartheta_{0} t}+b x_{\vartheta_{0}^{2} t}\right]+\varepsilon\left|\vartheta_{\tau_{\varepsilon}}^{*}-\vartheta_{0}\right|^{1 / 2} O(1)
$$


and we can write

$$
\begin{aligned}
& \varepsilon^{-1} \mathrm{I}_{\tau_{\varepsilon}}\left(\vartheta_{\tau_{\varepsilon}}^{*}\right)^{-1} b^{2} \int_{\tau_{\varepsilon}}^{T} t\left[a x_{t}+b x_{\vartheta_{\tau_{\varepsilon}}^{*}} t\right]\left(X_{\vartheta_{0} t}-X_{\vartheta_{\tau_{\varepsilon}}^{*} t}\right) \mathrm{d} t \\
& =-\frac{\left(\vartheta_{\tau_{\varepsilon}}^{*}-\vartheta_{0}\right)}{\varepsilon} \mathrm{I}_{\tau_{\varepsilon}}\left(\vartheta_{\tau_{\varepsilon}}^{*}\right)^{-1} b^{2} \int_{\tau_{\varepsilon}}^{T} t^{2}\left[a x_{t}+b x_{\vartheta_{\tau_{\varepsilon}} t}\right]\left[a x_{t}+b x_{\vartheta_{0} t}\right] \mathrm{d} t \\
& \quad+\left|\vartheta_{\tau_{\varepsilon}}^{*}-\vartheta_{0}\right|^{1 / 2} O(1) \\
& =-\frac{\left(\vartheta_{\tau_{\varepsilon}}^{*}-\vartheta_{0}\right)}{\varepsilon}\left[1+O\left(\vartheta_{\tau_{\varepsilon}}^{*}-\vartheta_{0}\right)\right]+\left|\vartheta_{\tau_{\varepsilon}}^{*}-\vartheta_{0}\right|^{1 / 2} O(1) .
\end{aligned}
$$

Here by conditions of the Theorem 5

$$
\frac{\left(\vartheta_{\tau_{\varepsilon}}^{*}-\vartheta_{0}\right)^{2}}{\varepsilon}=\frac{\varepsilon^{2 \delta}}{\varepsilon} O(1)=o(1)
$$

Finally we have

$$
\varepsilon^{-1}\left(\vartheta_{\varepsilon}^{\star}-\vartheta_{0}\right)=\frac{b}{\mathrm{I}\left(\vartheta_{0}\right)} \int_{0}^{T} t\left[a x_{t}+b x_{\vartheta_{0}}\right] \mathrm{d} W_{t}+o(1) .
$$

It can be verified that we have the uniform on compacts $\mathbb{K} \subset \Theta$ convergence of moments too

$$
\varepsilon^{-2} \sup _{\vartheta_{0} \in \mathbb{K}} \mathbf{E}_{\vartheta_{0}}\left|\vartheta_{\varepsilon}^{\star}-\vartheta_{0}\right|^{2} \longrightarrow \sup _{\vartheta_{0} \in \mathbb{K}} \mathrm{I}\left(\vartheta_{0}\right)
$$

Therefore for any (small) $\nu>0$

$$
\lim _{\varepsilon \rightarrow 0} \varepsilon^{-2} \sup _{\left|\vartheta-\vartheta_{0}\right| \leq \nu} \mathbf{E}_{\vartheta}\left|\vartheta_{\varepsilon}^{\star}-\vartheta\right|^{2}=\sup _{\left|\vartheta-\vartheta_{0}\right| \leq \nu} \mathrm{I}(\vartheta)
$$

and

$$
\lim _{\nu \rightarrow 0} \sup _{\left|\vartheta-\vartheta_{0}\right| \leq \nu} \mathrm{I}(\vartheta)=\mathrm{I}\left(\vartheta_{0}\right)
$$

Hence Two-step MLE $\vartheta_{\varepsilon}^{\star}$ is asymptotically efficient (see (14)).

The procedure of estimation is the following: first we calculate the estimator of substitution $\bar{\vartheta}_{\tau_{\varepsilon}}$, then with the help of this estimator we construct the Two-step MDE $\vartheta_{\tau_{\varepsilon}}^{*}$, which has the "good rate" and can be used for construction of the Two-step MLE $\vartheta_{\varepsilon}^{\star}$. This last estimator is asymptotically equivalent to the MLE. 
Let us write together all estimators. The first one is estimator of substitution

$$
\bar{\vartheta}_{\tau_{\varepsilon}}=\frac{2}{(a+b) b \tau_{\varepsilon}^{2}}\left[X_{\tau_{\varepsilon}}-x_{0}-x_{0} \tau_{\varepsilon}(a+b)\right]-\frac{a}{b} .
$$

Then, using this estimator we calculate the Two-step MDE

$$
\vartheta_{\tau_{\varepsilon}}^{*}=\bar{\vartheta}_{\tau_{\varepsilon}}+Q_{\tau_{\varepsilon}}\left(\bar{\vartheta}_{\tau_{\varepsilon}}\right)^{-1} \int_{0}^{\tau_{\varepsilon}}\left[X_{t}-x_{t}\left(\bar{\vartheta}_{\tau_{\varepsilon}}\right)\right] \dot{x}_{t}\left(\bar{\vartheta}_{\tau_{\varepsilon}}\right) \mathrm{d} t
$$

Finally, we can calculate the Two-step MLE

$$
\vartheta_{\varepsilon}^{\star}=\vartheta_{\tau_{\varepsilon}}^{*}+\mathrm{I}_{\tau_{\varepsilon}}\left(\vartheta_{\tau_{\varepsilon}}^{*}\right)^{-1} b \int_{\tau_{\varepsilon}}^{T} t\left[a x_{t}+b x_{\vartheta_{\tau_{\varepsilon}}^{*}}\right]\left[\mathrm{d} X_{t}-\left(a X_{t}+b X_{\vartheta_{\tau_{\varepsilon}}^{*}}\right) \mathrm{d} t\right] .
$$

The advantage of this procedure is the absence of the problems of optimization like (7) and (8).

Remark. Note that it is possible to introduce the Two-step MLE-process $\vartheta_{t, \varepsilon}^{\star}, \tau_{\varepsilon}<t \leq T$, which can be used in the problems, where we need estimator for all $t \in(0, T]$. For example, such estimators are used in the approximation of the solutions of backward stochastic differential equations [16]. Here this estimator-process can be written as follows

$$
\vartheta_{t, \varepsilon}^{\star}=\vartheta_{\tau_{\varepsilon}}^{*}+\mathrm{I}_{\tau_{\varepsilon}}^{t}\left(\vartheta_{\tau_{\varepsilon}}^{*}\right)^{-1} b \int_{\tau_{\varepsilon}}^{t} s\left[a x_{s}+b x_{\vartheta_{\tau_{\varepsilon}}^{*} s}\right]\left[\mathrm{d} X_{s}-\left(a X_{s}+b X_{\vartheta_{\tau_{\varepsilon}}^{*} s}\right) \mathrm{d} s\right],
$$

where $t \in\left(\tau_{\varepsilon}, T\right]$ and

$$
\mathrm{I}_{\tau}^{t}(\vartheta)=b^{2} \int_{\tau}^{t} \dot{x}_{s}(\vartheta)^{2} \mathrm{~d} s
$$

It can be shown that

$$
\varepsilon^{-1}\left(\vartheta_{t, \varepsilon}^{\star}-\vartheta_{0}\right) \Longrightarrow \mathcal{N}\left(0, \mathrm{I}_{\tau}^{t}\left(\vartheta_{0}\right)^{-1}\right) .
$$

For the proof of this convergence we can use the proof of the Theorem 5, where we just put $T=t$. Morover, it can be shown that we have the weak convergence of the stochastic process $\eta_{t, \varepsilon}=\mathrm{I}_{\tau}^{t}(\vartheta)$ (see [18]). 


\subsection{Nonlinear scale-type equation}

We consider the following nonlinear SDE

$$
\mathrm{d} X_{t}=S\left(X_{\vartheta t}\right) \mathrm{d} t+\varepsilon \mathrm{d} W_{t}, \quad X_{0}=x_{0}, \quad 0 \leq t \leq T,
$$

where $\vartheta \in \Theta=(\alpha, \beta), 0<\alpha<\beta<1$. Suppose that the function $S(x)$ has four continuous bounded derivatives. Recall that this equation has a unique strong solution (see Theorem 4.6 in [19]).

The limit differential equation is

$$
\frac{\mathrm{d} x_{t}}{\mathrm{~d} t}=S\left(x_{\vartheta t}\right), \quad x_{0}, \quad 0 \leq t \leq T .
$$

Its solution $x_{t}=x_{t}(\vartheta)$ is a function of $\vartheta$.

The preliminary MDE $\bar{\vartheta}_{\varepsilon}$ for this model of observations is defined by the same equation as in (8). This estimator is consistent, asymptotically normal and the moments converge. The proof is similar to the proof of Theorem 1.

The Fisher information is

$$
\mathrm{I}(\vartheta)=\int_{0}^{T} t^{2} S^{\prime}\left(x_{\vartheta t}(\vartheta)\right)^{2} S\left(x_{\vartheta^{2} t}(\vartheta)\right)^{2} \mathrm{~d} t .
$$

The properties of the MLE were studied in [1], [12] (linear case) and [13]. It was shown that the MLE $\hat{\vartheta}_{\varepsilon}$ is consistent, asymptotically normal

$$
\varepsilon^{-1}\left(\hat{\vartheta}_{\varepsilon}-\vartheta_{0}\right) \Longrightarrow \mathcal{N}\left(0, \mathrm{I}\left(\vartheta_{0}\right)^{-1}\right)
$$

and asymptotically efficient. Its numerical calculation can have the same difficulties as in the case of observations (1).

To introduce the One-step MLE we recall the definition of the functions $H(\cdot)$ and $\Psi(\cdot)$ for this model:

$$
\begin{aligned}
H(t, \vartheta) & =t S^{\prime}\left(x_{\vartheta t}(\vartheta)\right) S\left(x_{\vartheta^{2} t}(\vartheta)\right), \\
\Psi(\vartheta) & =H(T, \vartheta) X_{T}-H(0, \vartheta) x_{0}-\int_{0}^{T} H_{t}^{\prime}(t, \vartheta) X_{t} \mathrm{~d} t .
\end{aligned}
$$

Note that the function $H(\cdot)$ and its derivatives are bounded on the set $[0, T] \times$ $\Theta$. Then we can define

$$
\vartheta_{\varepsilon}^{\star}=\bar{\vartheta}_{\varepsilon}+\mathrm{I}\left(\bar{\vartheta}_{\varepsilon}\right)^{-1}\left[\Psi\left(\bar{\vartheta}_{\varepsilon}\right)-\int_{0}^{T} t S^{\prime}\left(x_{\bar{\vartheta}_{\varepsilon} t}\left(\bar{\vartheta}_{\varepsilon}\right)\right) S\left(x_{t \bar{\vartheta}_{\varepsilon}^{2}}\left(\bar{\vartheta}_{\varepsilon}\right)\right) S\left(X_{\bar{\vartheta}_{\varepsilon}}\right) \mathrm{d} t\right] .
$$

This One-step MLE is consistent and asymptotically normal

$$
\varepsilon^{-1}\left(\vartheta_{\varepsilon}^{\star}-\vartheta_{0}\right) \Longrightarrow \mathcal{N}\left(0, \mathrm{I}\left(\vartheta_{0}\right)^{-1}\right) \text {. }
$$

The proof follows the same lines as the proof of the Theorem 2 . 


\section{Discussion}

The proposed result can be generalized on the slightly more general models. For example, the cases with observations

$$
\mathrm{d} X_{t}=\left[S_{1}\left(X_{t}\right)+S_{2}\left(X_{t-\vartheta}\right)\right] \mathrm{d} t+\varepsilon \sigma\left(t, X_{t}\right) \mathrm{d} W_{t}, X_{s}=x_{s}, s \leq 0,0 \leq t \leq T
$$

and

$$
\mathrm{d} X_{t}=S\left(\vartheta, t, X_{t-\vartheta}\right) \mathrm{d} t+\varepsilon \sigma\left(t, X_{t}\right) \mathrm{d} W_{t}, \quad X_{s}=x_{s}, s \leq 0, \quad 0 \leq t \leq T
$$

can be treated exactly as it was done above. Here the functions $S_{1}(\cdot), S_{2}(\cdot)$, $S(\cdot), \sigma(\cdot)$ are supposed to be deterministic and smooth.

Another model, which can be treated by a similar way is

$$
\mathrm{d} X_{t}=S\left(X_{t-f(\vartheta, t)}\right) \mathrm{d} t+\varepsilon \mathrm{d} W_{t}, X_{s}=x_{s}, s \leq 0,0 \leq t \leq T .
$$

Here $f(\vartheta, t) \geq 0$ is some known smooth function. If $f(\vartheta, t)=\vartheta$ and $f(\vartheta, t)=$ $t-\vartheta t$ then we obtain the models (1) and (29) respectively.

It is possible to study the models with multiple delays like

$$
\mathrm{d} X_{t}=\sum_{k=1}^{K} S_{k}\left(X_{t-\vartheta_{k}}\right) \mathrm{d} t+\varepsilon \mathrm{d} W_{t}, \quad X_{s}=x_{s}, s \leq 0, \quad 0 \leq t \leq T .
$$

In the last model the delays form a vector $\vartheta=\left(\vartheta_{1}, \ldots, \vartheta_{K}\right)$, where $\vartheta_{1}<$ $\vartheta_{2}<\ldots<\vartheta_{K}$ and the problem of estimation $\vartheta$ became more complicate but the construction of the One-step procedure can be realized using the corresponding modification of the given above procedure.

\section{Appendix}

We suppose that the function $S(x), x \in \mathcal{R}$ has two continuous bounded derivatives $\left|S^{\prime}(x)\right| \leq L,\left|S^{\prime \prime}(x)\right| \leq M$, therefore the equation (1) has a unique strong solution (see Theorem 4.6 in [19]). Moreover, we have the following estimates (with probability 1)

$$
\begin{aligned}
\mid X_{t} & -x_{t}\left|\leq \int_{0}^{t}\right| S\left(X_{s-\vartheta_{0}}\right)-S\left(x_{s-\vartheta_{0}}\right)|\mathrm{d} s+\varepsilon| W_{t} \mid \\
& \leq L \int_{0}^{t}\left|X_{s-\vartheta_{0}}-x_{s-\vartheta_{0}}\right| \mathrm{d} s+\varepsilon\left|W_{t}\right| \leq L \int_{0}^{t}\left|X_{s}-x_{s}\right| \mathrm{d} s+\varepsilon \sup _{0 \leq s \leq T}\left|W_{s}\right|
\end{aligned}
$$

Recall the well-known lemma. 
Lemma 2 [Gronwall-Bellman] Suppose that the function $V(t) \geq 0,0 \leq t \leq$ $T$ satisfies the inequality

$$
V(t) \leq A \int_{0}^{t} V(s) \mathrm{d} s+B
$$

where the constants $A>0, B>0$. Then

$$
V(t) \leq B e^{A T}
$$

By this lemma we can write

$$
\left|X_{t}-x_{t}\right| \leq \varepsilon C \widehat{W}_{T}
$$

where we denoted $\widehat{W}_{T}=\sup _{0 \leq s \leq T}\left|W_{s}\right|$.

For the second moment we have

$$
\begin{aligned}
\mathbf{E}_{S}\left|X_{t}-x_{t}\right|^{2} & \leq 2 L^{2} T \int_{0}^{t} \mathbf{E}_{S}\left|X_{s-\vartheta_{0}}-x_{s-\vartheta_{0}}\right|^{2} \mathrm{~d} s+2 \varepsilon^{2} T \\
& \leq 2 L^{2} T \int_{0}^{t} \mathbf{E}_{S}\left|X_{s}-x_{s}\right|^{2} \mathrm{~d} s+2 \varepsilon^{2} T
\end{aligned}
$$

and by the same lemma

$$
\mathbf{E}_{S}\left|X_{t}-x_{t}\right|^{2} \leq C \varepsilon^{2}
$$

Recall that for $s \leq 0$ we have $X_{s}-x_{s}=x_{0}-x_{0}=0$.

Let us write the formal derivative $X_{t}^{(1)}$ of $X_{t}$ w.r.t. $\varepsilon$, then we obtain the equation

$$
\mathrm{d} X_{t}^{(1)}=S_{x}^{\prime}\left(X_{t-\vartheta_{0}}\right) X_{t-\vartheta_{0}}^{(1)} \mathrm{d} t+\mathrm{d} W_{t}, \quad X_{s}^{(1)}=0, \text { for } s \leq 0, \quad 0 \leq t \leq T .
$$

The Gaussian process $\left.X_{t}^{(1)}\right|_{\varepsilon=0}=x_{t}^{(1)}\left(\vartheta_{0}\right)$ satisfies the linear equation

$$
\mathrm{d} x_{t}^{(1)}=S_{x}^{\prime}\left(x_{t-\vartheta_{0}}\right) x_{t-\vartheta_{0}}^{(1)} \mathrm{d} t+\mathrm{d} W_{t}, \quad x_{s}^{(1)}=0, \text { for } s \leq 0, \quad 0 \leq t \leq T .
$$

The proof of

$$
V_{\varepsilon}(t)=\mathbf{E}_{\vartheta_{0}}\left|\frac{X_{t}-x_{t}\left(\vartheta_{0}\right)}{\varepsilon}-x_{t}^{(1)}(\vartheta)\right|^{2} \rightarrow 0
$$


can be done using the standard technique based on Gronwall-Bellman lemma as follows. We write

$$
\begin{aligned}
v_{\varepsilon}(t)= & \frac{X_{t}-x_{t}\left(\vartheta_{0}\right)}{\varepsilon}-x_{t}^{(1)} \\
= & \int_{0}^{t}\left[\frac{S\left(X_{s-\vartheta_{0}}\right)-S\left(x_{s-\vartheta_{0}}\right)}{\varepsilon}-S^{\prime}\left(x_{s-\vartheta_{0}}\right) x_{t}^{(1)}\right] \mathrm{d} s \\
= & \int_{0}^{t}\left[S^{\prime}\left(\tilde{X}_{s-\vartheta_{0}}\right) \frac{X_{s-\vartheta_{0}}-x_{s-\vartheta_{0}}}{\varepsilon}-S^{\prime}\left(x_{s-\vartheta_{0}}\right) x_{t}^{(1)}\right] \mathrm{d} s \\
= & \int_{0}^{t} S^{\prime}\left(\tilde{X}_{s-\vartheta_{0}}\right)\left[\frac{X_{s-\vartheta_{0}}-x_{s-\vartheta_{0}}}{\varepsilon}-S^{\prime}\left(x_{s-\vartheta_{0}}\right) x_{t}^{(1)}\right] \mathrm{d} s \\
& \quad+\int_{0}^{t}\left[S^{\prime}\left(\tilde{X}_{s-\vartheta_{0}}\right)-S^{\prime}\left(x_{s-\vartheta_{0}}\right)\right] \mathrm{d} s,
\end{aligned}
$$

where $\left|\tilde{X}_{s-\vartheta_{0}}-x_{s-\vartheta_{0}}\right| \leq\left|X_{s-\vartheta_{0}}-x_{s-\vartheta_{0}}\right|$. Hence

$$
\begin{aligned}
\mathbf{E}_{\vartheta_{0}}\left|v_{\varepsilon}(t)\right|^{2} \leq L^{2} T \int_{0}^{t} \mathbf{E}_{\vartheta_{0}}\left|v_{\varepsilon}\left(s-\vartheta_{0}\right)\right|^{2} \mathrm{~d} s \\
+M^{2} T \int_{0}^{T} \mathbf{E}_{\vartheta_{0}}\left|X_{t-\vartheta_{0}}-x_{t-\vartheta_{0}}\right|^{2} \mathrm{~d} s
\end{aligned}
$$

and

$$
\begin{aligned}
V_{\varepsilon}(t) & \leq L^{2} T \int_{0}^{t} V_{\varepsilon}\left(s-\vartheta_{0}\right) \mathrm{d} s+M^{2} T^{2} C \varepsilon^{2} \\
& \leq L^{2} T \int_{0}^{t} V_{\varepsilon}(s) \mathrm{d} s+M^{2} T^{2} C \varepsilon^{2} .
\end{aligned}
$$

Therefore by Gronwall-Bellman lemma $V_{\varepsilon}(t) \rightarrow 0$.

Let us study the quantity $R_{\varepsilon}(t)=\mathbf{E}_{\vartheta_{0}}\left|X_{t}-x_{t}\left(\vartheta_{0}\right)\right|^{p}$, where $x_{t}\left(\vartheta_{0}\right)$ and $X_{t}$ are solutions of the equations (17) and (18) respectively. For the difference $r_{\varepsilon}(t)=X_{t}-x_{t}\left(\vartheta_{0}\right)$ we have the relation

$$
r_{\varepsilon}(t)=\int_{0}^{t}\left[a r_{\varepsilon}(s)+b r_{\varepsilon}\left(\vartheta_{0} s\right)\right] \mathrm{d} s+\varepsilon W_{t} .
$$

Therefore

$$
\begin{aligned}
\mathbf{E}_{\vartheta_{0}}\left|r_{\varepsilon}(t)\right|^{p} & \leq C T^{p-1}|a|^{p} \int_{0}^{t} \mathbf{E}_{\vartheta_{0}}\left|r_{\varepsilon}(s)\right|^{p} \mathrm{~d} s+C|b|^{p} \vartheta_{0}^{-1} \int_{0}^{\vartheta_{0} t} \mathbf{E}_{\vartheta_{0}}\left|r_{\varepsilon}(s)\right|^{p} \mathrm{~d} s \\
& +C \varepsilon^{p} T^{\frac{p}{2}} \\
& \leq\left[C T^{p-1}|a|^{p}+C|b|^{p} \vartheta_{0}^{-1}\right] \int_{0}^{t} \mathbf{E}_{\vartheta_{0}}\left|r_{\varepsilon}(s)\right|^{p} \mathrm{~d} s+C \varepsilon^{p} T^{\frac{p}{2}}
\end{aligned}
$$


and

$$
R_{\varepsilon}(t) \leq\left[C T^{p-1}|a|^{p}+C|b|^{p} \vartheta_{0}^{-1}\right] \int_{0}^{t} \mathbf{E}_{\vartheta_{0}}\left|R_{\varepsilon}(s)\right|^{p} \mathrm{~d} s+C \varepsilon^{p} T^{\frac{p}{2}} .
$$

Now by Gronwall-Bellman lemma we obtain the estimate

$$
\mathbf{E}_{\vartheta_{0}}\left|X_{t}-x_{t}\left(\vartheta_{0}\right)\right|^{p} \leq C \varepsilon^{p}
$$

with the corresponding constant $C>0$.

\section{Acknowledgments}

This research was supported by RSF project no 20-61-47043.

\section{References}

[1] Apoyan G.T. (1986) Parameter estimation of non differentiable trend coefficient. (in Russian) Uchen. Zap. Erevan Univ. 1, 33-62.

[2] Bandrivskyy, A., Luchinsky, D.G., McClintock, P.V.E., SmeLYAnskiy, V. N. And Stefanovska, A. (2005) Inference of systems with delay and applications to cardiovascular dynamics Stochastics and Dynamics, 5, 2, 321-331.

[3] Frank, T.D., Friedrich, R. And Beek, P.J. (2005) Time series analysis of multivariate time-delayed systems with noise: applications to laser physics and human movement Stochastics and Dynamics, 5, 2, 297-306.

[4] Freidlin M.I. And Wentsell A.D. (1984) Random Perturbations of Dynamical Systems, Springer, New York.

[5] Griebel, T. (2017) The Pantograph Equation in Quantum Calculus. Missouri University of Science and Technology, Masters Theses. 7644. https://scholarsmine.mst.edu/masters_theses/7644

[6] Gushchin, A.A. And KüChler, U. (1999) Asymptotic inference for a linear stochastic differential equation with time delay. Bernoulli 5, 6, 1059-1098.

[7] Hauptmann, C., Popovych, O. And Tass, P.A. (2005) Multisite coordinated delayed feedback for an effective desynchronization of neuronal networks Stochastics and Dynamics, 5, 2, 307-319. 
[8] Ibragimov I.A. And Has'minskin R.Z. (1981) Statistical Estimation: Asymptotic Theory, Springer, Berlin.

[9] Kuang, Y. (1993) Delay Differential Equations: With Applications in Population Dynamics, Academic Press, London.

[10] KüChler, U., Kutoyants, Yu. A. (2000) Delay estimation for some stationary diffusion-type processes, Scandinavian Journal of Statistics, 27, 3, 405-414.

[11] KÜChler, U. Mensch, B. (1992) Langevins stochastic differential equation extended by a time-delayed term, Stochastics and Stochastic Reports, 40, 23-42.

[12] Kutoyants, Yu. A. (1988). An example of estimating a parameter of a nondifferentiable drift coefficient. Theory Probab. Appl., 33, 1, 175-179.

[13] Kutoyants, Yu. A. (1994) Identification of Dynamical Systems with Small Noise. Kluwer, Dordrecht.

[14] Kutoyants, Yu. A. (2004) Statistical Inference for Ergodic Diffusion Processes. Springer, London.

[15] Kutoyants, Yu. A. (2005) On delay estimation for stochastic differential equations. Stochastics and Dynamics, 5, 2, 333-342.

[16] Kutoyants, Yu.A. (2014) Approximation of the solution of the backward stochastic differential equation. Small noise, large samples and high frequency cases. Proceedings of the Steklov Institute of Mathematics, 287, 133-154.

[17] Kutoyants, Yu.A. (2017) On the multi-step MLE-process for ergodic diffusion, Stochastic Processes and their Applications, 127, 2243-2261.

[18] Kutoyants, Y.A. And Zhou, L. (2014) On approximation of the backward stochastic differential equation. J. Stat. Plann. Infer. 150, 111-123.

[19] Liptser, R. S. And Shiryaev, A. N. (2001). Statistics of Random Processes. 2-nd ed., Vol. 1, Springer, New York.

[20] MAO, X. (1994) Exponential stability of stochastic differential equations, Marcel Dekker, New York. 
[21] MAO, X. (2005) Delay population dynamics and environmental noise. Stochastics and Dynamics, 5, 2, 149-162.

[22] Myshkis, A.D. (1951) Linear Differential Equations with Delayed Argument. (in Russian) Nauka, Moscow.

[23] Ockendon, J.R., TAyler, A.B. (1971) The dynamics of a current collection system for an electric locomotive. In Proceedings of the Royal Society of London A: Mathematical, Physical and Engineering Sciences, $322,447-468$.

[24] Ohira, T., Yamane, T. (2000) Delayed stochastic systems. Physical Review. E, Statistical Physics, Plasmas, Fluids and Related Interdisciplinary Topics 61, 2, 1247-1257.

[25] Rosinberg, M.L., Taruus, G. and Munakata, T. (2018) Influence of time delay on information exchanges between coupled linear stochastic systems. Physical Review. E, 98, 3, 032130. 